\title{
Recursive allocations and wealth distribution with multiple goods: Existence, survivorship, and dynamics
}

\author{
R. Colacito \\ Kenan-Flagler Business School, University of North Carolina at Chapel Hill and NBER \\ M. M. Croce \\ Finance Department, Bocconi University, CEPR, and NBER \\ ZHAO LiU \\ Department of Economics, Duke University
}

\begin{abstract}
We characterize the equilibrium of a complete markets economy with multiple agents featuring a preference for the timing of the resolution of uncertainty. Utilities are defined over an aggregate of two goods. We provide conditions under which the solution of the planner's problem exists, and it features a nondegenerate invariant distribution of Pareto weights. We also show that perturbation methods replicate the salient features of our recursive risk-sharing scheme, provided that higher-order terms are included.
\end{abstract}

KEYWORDs. Recursive preferences, multiple agents, equilibrium.

JEL CLASSification. C62, F37.

\section{INTRODUCTION}

In the context of single-agent economies, recursive preferences have become increasingly relevant for the analysis of issues at the forefront of the macro-finance agenda (see, among others, Hansen and Sargent (1995), Tallarini (2000), Bansal and Yaron (2004), and Backus, Routledge, and Zin (2005)). In models populated by multiple agents, in contrast, the adoption of recursive preferences is less common, as it produces a key challenge in the characterization of the risk-sharing dynamics.

With recursive preferences, in fact, optimal allocations are a function not only of aggregate endowment, but also of a possibly time-varying distribution of wealth. As documented by Anderson (2005), in a one-good economy in which agents have risk-sensitive preferences there is typically a tension between ensuring that a nondegenerate distribution of wealth exists and having interesting heterogeneity across agents. The same

R. Colacito: ric@unc.edu

M. M. Croce: mmc287@gmail.com

Zhao Liu: zhao. liu@duke.edu

We thank seminar participants at the 2009 Meeting of the Society for Economic Dynamics in Istanbul and at the 2010 Meeting of American Economic Association in Atlanta. We are grateful to Lars Hansen, Thomas Philippon, Tom Sargent, and the participants at the $2010 \mathrm{PhD}$ mini-course on Asset Pricing and Risk Sharing with Recursive Preferences at NYU, which was partly based on this paper. All errors remain our own.

(c) 2019 The Authors. Licensed under the Creative Commons Attribution-NonCommercial License 4.0. Available at http://qeconomics.org. https://doi.org/10.3982/QE457 
paper documents that this tension can be relaxed if multiple preference parameters are changed simultaneously and agents have power-reward functions with risk aversion between zero and one.

In this paper, we overcome these challenges by focusing on an economy with multiple goods. We show that rich dynamics of Pareto optimal allocations are obtained even in the case in which all agents share the same risk-sensitivity parameter and have logarithmic period reward functions, provided that they feature a different degree of preference for one of the two goods. Furthermore, we provide conditions under which a nondegenerate ergodic distribution of Pareto weights exists. This is the case in which every agent in the economy has a strictly positive wealth in the long run.

An agent with recursive preferences is willing to trade off expected utility for higher conditional moments of future utility. In a world with a Cobb-Douglas aggregate over multiple goods, the intensity of this trade-off is stronger for agents that consume a large share of aggregate resources, that is, agents with high Pareto weights. For example, as shown in a simple two-period model, when agent 1 has a high initial share of resources, she will have a strong incentive to buy insurance from agent 2 to mitigate future utility uncertainty.

In equilibrium, under a preference for early resolution of uncertainty, agent 1 accepts a reduction in her expected average share of resources (i.e., her Pareto weight is expected to decline) in exchange for a reduction of future utility variance. This tradeoff between expected utility and conditional volatility of future utility results in a welldefined invariant distribution of Pareto weights.

Several authors have documented the theoretical properties of one-good versions of the economy analyzed in this paper (Lucas and Stokey (1984), Ma (1993), and Kan (1995)). In particular, Anderson (2005) shows that in an economy with heterogenous agents and recursive preferences it is difficult to ensure the existence of a nondegenerate ergodic distribution of wealth, unless very extreme forms of heterogeneity are considered (see, e.g., Backus, Routledge, and Zin (2008)).

Our focus on the case of a consumption aggregate of multiple goods resolves these problems, and it is important in many economic applications. In a closed economy, we may think of agents featuring different propensities across commodities produced by different firms or sectors. In an open economy, it is common to assume that consumers located in different countries are biased toward the consumption of the domestically produced good (see, e.g., Tretvoll (2018)). The economy analyzed in this paper provides the foundations for the international macro-finance model in Colacito and Croce (2013). In related work, Backus, Coleman, Ferriere, and Lyon (2016) showed that the endogenous variation in Pareto weights in the type of economies that we consider can be interpreted as wedges from the perspective of a frictionless model with additive preferences.

Colacito and Croce (2012) applied the results in this manuscript to the heterogeneous-beliefs literature (among others, see Kubler and Schmedders (2012) and Tsyrennikov (2012)). ${ }^{1}$ They show that consumption home bias is isomorphic to endogenous disagreement about the fundamentals of the economy. Under the conditions explored in our paper, the ergodic distribution of wealth is nondegenerate, despite the

\footnotetext{
${ }^{1}$ Specifically, Colacito and Croce (2012) interpreted the preferences used in this manuscript as describing a concern for model misspecification, according to the definition of Hansen and Sargent (2008). This results
} 
existence of endogenous heterogenous beliefs. For a detailed study of both, the survivorship and risk sharing in economies populated by recursive agents with exogenous heterogeneous beliefs see Borovička (2018).

From a computational point of view, the characterization of the risk-sharing arrangement with recursive preferences poses additional challenges, as the state space includes the relative wealth of the agents, which in turn, depends on the continuation utilities. We compare a global method that uses value-function iterations and a perturbation-based approach and document that a first-order Taylor expansion about the stochastic steady state of the economy is not appropriate for capturing the dynamics of the economy. This approximation severely deteriorates in regions distant from the steady state, and it produces a counterfactual limiting wealth distribution in which either agent may find herself with zero wealth with probability one. Higher-order approximations are necessary not only to provide a better period-by-period characterization of the dynamics of the model, but also to capture the stationarity of the model. These findings are consistent with the analysis of Anderson, Hansen, and Sargent (2012) and Pohl, Schmedders, and Wilms (2017).

Additionally, we show that our setting produces endogenous time variation in higher-order conditional moments of consumption, and hence it offers general equilibrium foundations for the analyses of Bansal, Kiku, Shaliastovich, and Yaron (2014), Kuehn and Boguth (2013), Colacito, Ghysels, Meng, and Siwarasit (2016), and Segal, Shaliastovich, and Yaron (2015).

We also study important extensions of our benchmark model by considering the case in which agents have intertemporal elasticities of substitution different from 1 and endowment shocks that are persistent. This means that our analysis can be informative for the growing body of the literature that has explored the macro-finance implications of Epstein and Zin (1989) preferences (see, e.g., Bansal et al. (2014)). Furthermore, we show that the introduction of a moderate degree of heterogeneity in the calibration of the two countries may still result in a well-defined ergodic distribution of wealth in equilibrium. This is relevant for the application of our study to economies in which, for example, investors in different countries face a heterogeneous degree of fundamental risk in their endowments or productivities.

Baker and Routledge (2017) studied an economy similar to the one analyzed in this paper. Like us, they consider the risk-sharing arrangement between two agents with recursive preferences defined over a Cobb-Douglas aggregate of two goods: oil and a general consumption good. Since the main focus of their paper is matching the price of oil and related futures contracts, they rely on asymmetric calibrations of the two agents. This choice typically results in the survivorship of only one of the two agents in the economy. In this respect, the results that we provide in Section 5, in which we relax the symmetry of the calibration, are informative for the general class of model that they consider.

in agent-specific distorted conditional distributions of the endowment processes. Since each probability depends on the utility of a specific agent, when preferences feature heterogenous bias across goods, agents disagree on the transition probabilities across states of the world. 
Our paper is organized as follows. In Section 2, we provide the setup of our benchmark economy, featuring unit intertemporal elasticity of substitution and i.i.d. shocks. In Section 3, we discuss the main intuitions of our framework in the context of a simple two periods model and provide the set of conditions under which a nondegenerate limiting distribution of Pareto weights exists in the infinite horizon setting. In Section 4, we compare a numerical solution of the model obtained via value function iteration with first- and higher-order approximations. In Section 5, we present the results of several generalized versions of our baseline setup. Section 6 concludes the paper.

\section{Setup of THE ECONOMY}

In this section, we describe the assumptions that we use in the benchmark version of our model. For the purpose of simplifying the analytical proofs and the intuitions of the model, in our benchmark we assume that the intertemporal elasticity of substitution is equal to one and that the two countries share a symmetrical calibration. In Section 5, we use simulations to show that our results apply to more general settings.

The following three assumptions about preferences, consumption, and endowments will be retained throughout the rest of the paper.

Assumption 1 (Preferences). Let there be two agents, indexed by 1 and 2, whose preferences are recursively defined as

$$
U_{i}\left(c_{i}, q_{i}\right)=\left(1-\delta_{i}\right) \log c_{i}+\delta_{i} \theta_{i} \log \sum_{z^{\prime}} \pi\left(z^{\prime}\right) \exp \left\{\frac{q_{i}\left(z^{\prime}\right)}{\theta_{i}}\right\}, \quad \forall i \in\{1,2\},
$$

where $q_{i}\left(z^{\prime}\right)$ gives the utility remaining from the next period on when next-period's state is $z^{\prime}$. For each agent $i, \theta_{i}<0$.

This class of preferences can be interpreted in several ways. First, they correspond to the case of risk-sensitive preferences studied by, among others, Hansen and Sargent (1995), Tallarini (2000), and Anderson (2005). Second, they coincide with a logtransformation of Epstein and Zin (1989) preferences in the case in which the intertemporal elasticity of substitution is equal to one. In this case, the risk-sensitive parameter, $\theta$, is related to risk aversion, $\gamma$, by imposing

$$
\theta=\frac{1}{1-\gamma}
$$

In this paper, we focus on a discrete time setting as opposed to the continuous time approach of Epstein (1987), Duffie, Geoffard, and Skiadas (1994), Geoffard (1996), and Dumas, Uppal, and Wang (2000).

Since these preferences depart from the expected utility case, higher moments of continuation utilities matter for the determination of optimal risk sharing. As as example, if continuation utilities $q_{i}\left(z^{\prime}\right)$ are normally distributed, the functional form in (1) can be written as

$$
U_{i}\left(c_{i}, q_{i}\right)=\left(1-\delta_{i}\right) \log c_{i}+\delta_{i} E_{i}\left(q_{i}\right)+\frac{\delta_{i}}{2 \theta_{i}} V_{i}\left(q_{i}\right), \quad \forall i \in\{1,2\},
$$


where

$$
\begin{aligned}
& E_{i}\left(q_{i}\right)=\sum_{z^{\prime}} \pi\left(z^{\prime}\right) q_{i}\left(z^{\prime}\right), \\
& V_{i}\left(q_{i}\right)=\sum_{z^{\prime}} \pi\left(z^{\prime}\right)\left[q_{i}\left(z^{\prime}\right)-\left(\sum_{z^{\prime}} \pi\left(z^{\prime}\right) q_{i}\left(z^{\prime}\right)\right)\right]^{2}
\end{aligned}
$$

are the conditional mean and variance of the continuation utility, respectively.

Although we will work with the general specification in (1), equation (2) is instructive, since it intuitively shows that when the parameters $\theta_{i}$ 's are less than zero, the risksharing scheme must account for an efficient endogenous trade-off between utility level and utility variance.

As the dynamics of second-order moments are crucial for characterizing the equilibrium of the model, in Section 4 we also assess the accuracy of several approximations based on how well they can account for the dynamics of volatilities.

Assumption 2 (Consumption Bundles). Let consumption $c_{i}$ be an aggregate of two goods, $x_{i}$ and $y_{i}$. Specifically, let

$$
c_{i}=\left(x_{i}\right)^{\lambda_{i}}\left(y_{i}\right)^{1-\lambda_{i}}
$$

be the consumption bundle, with $\lambda_{1}>1 / 2$ and $\lambda_{2}<1 / 2$ so that the two agents have a bias for different goods.

This assumption generalizes the one-good framework studied by Anderson (2005), which obtains as the special case in which $\lambda_{i}=1 / 2, \forall i$, that is, the case in which there is no preference bias across goods, and hence we are effectively in a one-good economy.

The next assumption pertains to the endowment process and is common to Anderson (2005).

Assumption 3 (Endowments). The endowment of the two goods follows a first-order time-homogenous Markov process $\left(z_{0}, z_{1}, \ldots\right)$ which takes values in a finite set $\mathcal{N}=$ $\{1, \ldots, n\}$. The aggregate supply of the two goods at time $t$ is such that $0<X_{t}=X\left(z_{t}\right)<\infty$, and $0<Y_{t}=Y\left(z_{t}\right)<\infty$.

Finally, we need to make sure that the preference parameters are chosen so that the utility recursion converges:

Assumption 4 (Contraction). The parameters $\left\{\lambda_{i}, \gamma_{i}, \delta_{i}\right\}$ are such that the right-hand side of equation (1) has a modulus smaller than one, $\forall i=\{1,2\}$.

\section{Recursive planner's problem}

Let $\log W_{i}^{*}\left(z, c_{i},\left\{q_{i, z^{\prime}}\right\}_{z^{\prime}}\right)$ be the right-hand side of equation (1). Given the conditions specified by Ma (1993) and Ma (1996), the social planner's value function, denoted as 
$Q_{p}\left(z, \mu_{1}\right): \mathcal{N} \times[0,1] \rightarrow R$, satisfies the following functional equation proposed by Lucas and Stokey (1984) and Kan (1995):

$$
Q_{p}\left(z, \mu_{1}\right)=\max _{\left\{x_{i}, y_{i}, q_{i, z^{\prime}}\right\}_{i \in\{1,2\}, z^{\prime} \in \mathcal{N}}} \sum_{i=1}^{2} \mu_{i} \log W_{i}^{*}\left(z, c_{i},\left\{q_{i, z^{\prime}}\right\}_{z^{\prime}}\right)
$$

subject to

$$
\begin{aligned}
\mu_{2} & =1-\mu_{1}, \\
0 & \leq x_{1} \leq X(z), \quad 0 \leq x_{2} \leq X(z)-x_{1}, \\
0 & \leq y_{1} \leq Y(z), \quad 0 \leq y_{2} \leq Y(z)-y_{1}, \\
c_{i} & =\left(x_{i}\right)^{\lambda_{i}}\left(y_{i}\right)^{1-\lambda_{i}}, \quad \forall i=\{1,2\}, \\
0 & \leq \min _{\mu_{1}\left(z^{\prime}\right) \in[0,1]} Q_{p}\left(z^{\prime}, \mu_{1}\left(z^{\prime}\right)\right)-\mu_{1}\left(z^{\prime}\right) q_{1, z^{\prime}}-\left(1-\mu_{1}\left(z^{\prime}\right)\right) q_{2, z^{\prime}}, \quad \forall z^{\prime} \in \mathcal{N} .
\end{aligned}
$$

\section{Differentiability and first-order conditions}

Let the ratio of the Pareto weights be defined as

$$
S=\frac{\mu_{1}}{\mu_{2}}=\frac{\mu_{1}}{1-\mu_{1}} .
$$

Let $U_{i}(z, S), i=1,2$, denote the utility function of agent $i$ evaluated at the optimum when the exogenous state is $z$, and $S \in(0, \infty)$. On a consumption path that is bounded away from zero, $U_{i}(z, S)$ is differentiable (see Kan (1995) and Anderson (2005)) and

$$
\frac{d U_{i}}{d \mu_{i}}>0, \quad \mu_{1} \in(0,1), \mu_{2}=1-\mu_{1} .
$$

On a consumption path that is bounded away from zero for both agents, $Q_{p}\left(s, \mu_{1}\right)$ is also differentiable with respect to $\mu_{1} \in(0,1)$. The optimality condition in equation (5) implies that

$$
\begin{aligned}
& \frac{d Q_{p}}{d \mu_{1}}\left(z, \mu_{1}\right)=U_{1}(z, S)-U_{2}(z, S), \quad \mu_{1} \in(0,1), \\
& \frac{d^{2} Q_{p}}{d \mu_{1}^{2}}\left(z, \mu_{1}\right)=\frac{d U_{1}}{d \mu_{1}}(z, S)+\frac{d U_{2}}{d \mu_{2}}(z, S)>0 .
\end{aligned}
$$

Therefore, $Q_{p}\left(s, \mu_{1}\right)$ is strictly convex with respect to $\mu_{1}$, as in Lucas and Stokey (1984). This is relevant because it implies that the unique optimal policy of the planner can be characterized using first-order conditions.

According to the first-order conditions, for a given $S$, the optimal allocation of goods satisfies the following system of equations common to all static Pareto problems with two goods and two agents:

$$
\left(1-\delta_{1}\right) \frac{\partial \log c_{1}}{\partial x_{1}} \cdot S=\left(1-\delta_{2}\right) \frac{\partial \log c_{2}}{\partial x_{2}}
$$




$$
\begin{gathered}
\left(1-\delta_{1}\right) \frac{\partial \log c_{1}}{\partial y_{1}} \cdot S=\left(1-\delta_{2}\right) \frac{\partial \log c_{2}}{\partial y_{2}}, \\
X=x_{1}+x_{2}, \quad Y=y_{1}+y_{2} .
\end{gathered}
$$

The optimal dynamic adjustment of the ratio of the pseudo-Pareto weights is then given by

$$
S^{\prime}=S \cdot \mathcal{M}\left(z^{\prime}, S^{\prime}\right)
$$

where

$$
\mathcal{M}\left(z^{\prime}, S^{\prime}\right) \equiv \frac{\delta_{1} \exp \left\{U_{1}\left(z^{\prime}, S^{\prime}\right) / \theta_{1}\right\}}{\sum_{z^{\prime}} \pi\left(z^{\prime}\right) \exp \left\{U_{1}\left(z^{\prime}, S^{\prime}\right) / \theta_{1}\right\}} \cdot \frac{\sum_{z^{\prime}} \pi\left(z^{\prime}\right) \exp \left\{U_{2}\left(z^{\prime}, S^{\prime}\right) / \theta_{2}\right\}}{\delta_{2} \exp \left\{U_{2}\left(z^{\prime}, S^{\prime}\right) / \theta_{2}\right\}} .
$$

Equation (9) determines the dynamics of the ratio of the Pareto weights and implicitly generates a continuous function that we denote by $f_{S}\left(z^{\prime}, \cdot\right):[0,+\infty) \rightarrow[0,+\infty)$ :

$$
S^{\prime}=f_{S}\left(z^{\prime}, S\right)
$$

Characterizing the planner's problem through first-order conditions is useful because it allows us to represent the planner's problem in (4) as a simple system of firstorder stochastic difference equations, namely (1), (3), (8), and (9). In the next section, we use perturbation methods to solve our dynamic system of equations.

\section{Relative price of the two goods}

The relative price of the two goods, $p$, is the equilibrium marginal rate of substitution across goods

$$
p=\frac{\left(1-\lambda_{1}\right)}{\lambda_{1}} \cdot \frac{x_{1}}{y_{1}} .
$$

Given the optimal allocations of $x_{1}$ and $y_{1}$, we can write the relative price as

$$
p=\tilde{p} \cdot \frac{X}{Y}
$$

where

$$
\tilde{p} \equiv\left(1-\lambda_{1}\right) / \lambda_{1} \cdot\left[1+S \cdot \frac{\left(1-\lambda_{1}\right)}{\left(1-\lambda_{2}\right)}\right] /\left[1+S \cdot \frac{\lambda_{1}}{\lambda_{2}}\right]
$$

When the supply of good $X$ relative to good $Y$ is high, the price of good $Y$ increases for two reasons. First, the last term $(X / Y)$ directly affects the relative price. This channel would be at work even for $\lambda_{1}=1 / 2$, in which case $\tilde{p}=1$, and $p=X / Y$. Second, since $\lambda_{1}>\lambda_{2}$ it follows that

$$
\frac{\partial \tilde{p}}{\partial S}=-\frac{1-\lambda_{1}}{\lambda_{1}} \cdot \frac{\lambda_{2}}{1-\lambda_{2}} \cdot \frac{\left(\lambda_{1}-\lambda_{2}\right)}{\left(\lambda_{2}+\lambda_{1} \cdot S\right)^{2}}<0 .
$$


This means that the price will further increase as long as the ratio of Pareto weights $(S)$ declines when $X / Y$ is large (we prove this statement formally in Proposition 1). Equivalently, the price of good $Y$ is large whenever its supply is low. This effect is magnified in the context of our model since $\lambda_{1}>1 / 2, \lambda_{2}<1 / 2$, and the ratio of Pareto weights can move away from a symmetric wealth distribution. This enhanced price adjustment allows agent 2 to purchase a larger share of resources whenever the supply of its most preferred good is low.

\section{Share of world consumption (SWC)}

We note that under our Cobb-Douglas aggregator across goods, the relative share of world consumption of agent $1, S W C$, evolves as follows:

$$
S W C=\frac{x_{1}+p y_{1}}{X+p Y}=\frac{S}{1+S}=\mu_{1} .
$$

According to equations (8) and (11), the Pareto weight of agent 1 has a simple economic interpretation, namely, the relative size of consumption allocated to agent 1.

\section{Symmetry}

So far, we have not imposed any specific assumptions on the conditional probability of the Markov chain governing the supply of the two goods, nor have we imposed any special restrictions on the preference parameters of our agents. In order to have a wellspecified problem, all we need is that Assumptions 1-4 hold. In what follows, however, we list further restrictions that are necessary to analytically characterize the main properties of the optimal risk-sharing policy of the planner. These conditions impose symmetry and are sufficient, but in many cases not necessary, for the existence of a stationary distribution. In the next section, we relax many of these assumptions.

Assumption 5 (Symmetrical Preferences). Let the preference parameters $\delta_{i}$ and $\theta_{i}$ be identical $\forall i \in\{1,2\}$. Let the consumption-bundle's parameters be symmetrical across agents, that is, $\lambda_{1}=1-\lambda_{2}>1 / 2$.

Assumption 6 (Balanced Endowment Space). Let the support of the endowment of good $X$ be given by the vector $H=\left[h_{1}, h_{2}, \ldots, h_{N}\right]$. Let the support of the endowment of good $Y$ be $H$ as well. The endowments of the two goods take values in the finite set $\mathcal{N}$, given by all possible pairwise permutations of $H$. We refer to $\mathcal{N}$ as a balanced endowment space.

Definition 1 (Symmetric States). Let the states $z_{i}, z_{-i} \in \mathcal{N}$ be such that $z_{i}=\left\{X_{i}=\right.$ $\left.X(i), Y_{i}=Y(i)\right\}$ and $z_{-i}=\left\{X_{-i}=Y(i), Y_{-i}=X(i)\right\}$. Then $z_{i}$ and $z_{-i}$ are symmetric states.

Finally, just to simplify our proof, we focus on a setting with i.i.d. shocks and on symmetric probability distributions. 
Assumption 7 (I.i.d. Case). Assume that $\pi\left(z^{\prime} \mid z\right)=\pi\left(z^{\prime}\right)>0, \forall z, z^{\prime} \in \mathcal{N}$.

Assumption 8 (Symmetric Probabilities). Let the states $z_{i}, z_{-i} \in \mathcal{N}$ be symmetric. Then $\pi\left(z_{i}\right)=\pi\left(z_{-i}\right)$.

\section{Characterizing the distribution of Pareto weights}

In this section, we show the main properties of the Pareto weights under our recursive scheme in two settings. We start with a simplified two-period model for which we have a closed-form approximate solution. We then provide results for our infinite-horizon economy.

\subsection{Recursive risk-sharing in a two-period model}

The goal of this section is to illustrate the role of the income and substitution effects as a function of the initial Pareto weights. To highlight the key features of the model, we focus on the special case in which symmetry applies, consistent with the assumptions employed for our main propositions. In Appendix A in the Supplemental Material (available in the supplementary file on the journal website, http://qeconomics.org/ supp/457/code_and_data.zip), we study a two-period version of the planner's problem detailed in the system of equations (4)-(5) and provide a general solution which allows for asymmetries across the two agents.

Specifically, consider the following Pareto problem:

$$
\max _{\left\{X_{1}^{1}, X_{1}^{2}, Y_{1}^{1}, Y_{1}^{2}\right\}} \mu_{0} \cdot \theta \log E_{0}\left[\exp \left\{\frac{u_{1}^{1}}{\theta}\right\}\right]+\left(1-\mu_{0}\right) \cdot \theta \log E_{0}\left[\exp \left\{\frac{u_{1}^{2}}{\theta}\right\}\right],
$$

subject to the following conditions:

$$
\begin{aligned}
u_{1}^{i} & =\log \left(C_{1}^{i}\right), \quad \forall i=\{1,2\}, \\
C_{1}^{1} & =\left(X_{1}^{1}\right)^{\lambda}\left(Y_{1}^{1}\right)^{(1-\lambda)}, \quad C_{1}^{2}=\left(X_{1}^{2}\right)^{(1-\lambda)}\left(Y_{1}^{2}\right)^{\lambda},
\end{aligned}
$$

and the following resource constraints:

$$
X_{1}^{1}+X_{1}^{2}=e^{\xi}, \quad Y_{1}^{1}+Y_{1}^{2}=e^{-\xi}, \quad \xi \sim N\left(0, \sigma^{2}\right),
$$

where, for simplicity, we are assuming that the endowments of the two goods are perfectly negatively correlated. Let $\bar{s}=\log \left(\mu_{0} /\left(1-\mu_{0}\right)\right)$ and let $s$ be the log-ratio of the pseudo-Pareto weights at time 1 . We use this simple setup to illustrate three key features of this class of models.

Income and substitution effects We show in Appendix A in the Supplemental Material (available in a supplementary file on the journal website, http://qeconomics.org/supp/ 457/code_and_data.zip) that the equilibrium utility functions can be written as

$$
u_{1}^{i}=\bar{u}_{1}^{i}+\lambda_{u_{1}^{i}}^{\xi} \cdot \xi, \quad \forall i=\{1,2\}
$$


where

$$
\begin{aligned}
& \lambda_{u_{1}^{1}}^{\xi}=\underbrace{(2 \lambda-1)}_{>0}[1+\underbrace{\frac{2 \beta(\bar{s})}{\theta-\beta(\bar{s})\left(1+e^{\bar{s}}\right)}}_{<0}], \text { and } \\
& \lambda_{u_{1}^{2}}^{\xi}=-\underbrace{(2 \lambda-1)}_{>0}[1+\underbrace{\frac{2 \beta(\bar{s}) e^{\bar{s}}}{\theta-\beta(\bar{s})\left(1+e^{\bar{s}}\right)}}_{<0}]
\end{aligned}
$$

with $\beta(\bar{s})$ defined as the following nonnegative, and monotonically decreasing function of $\bar{s}$ :

$$
\beta(\bar{s})=\left[\frac{\lambda(1-\lambda)}{(1-\lambda)+\lambda e^{\bar{s}}}+\frac{\lambda(1-\lambda)}{\lambda+(1-\lambda) e^{\bar{s}}}\right] .
$$

Without loss of generality, let us focus on the response of agent 1's utility to an endowment shock $\xi$, which is captured by the coefficient $\lambda_{u_{1}^{1}}^{\xi}$. A positive endowment effect, measured by $(2 \lambda-1)$, determines an increase in the utility that is proportional to the degree of preference for good $X$. In a single-agent economy, that is, for $\bar{s} \rightarrow+\infty$, this is the only effect relevant for the dynamics of future utilities. In a multiple-agent economy, an additional negative redistribution effect captures the reallocation of resources that takes place by virtue of risk sharing. This effect depends on $\beta(\bar{s})$, and hence it declines with the original ratio of Pareto weights, $\bar{s}$.

Redistribution of resources Using the equilibrium utilities in (12), it is easy to show that the transition dynamics of the logarithm of the ratio of the Pareto weights in (9) becomes

$$
\begin{aligned}
s-\bar{s} & =\frac{u_{1}^{1}}{\theta}-\frac{u_{1}^{2}}{\theta}-\left[\frac{E_{0}\left(u_{1}^{1}\right)}{\theta}+\frac{\operatorname{Var}_{0}\left(u_{1}^{1}\right)}{2 \theta^{2}}\right]+\left[\frac{E_{0}\left(u_{1}^{2}\right)}{\theta}+\frac{\operatorname{Var}_{0}\left(u_{1}^{2}\right)}{2 \theta^{2}}\right] \\
& =\left[\frac{\operatorname{Var}_{0}\left(u_{1}^{2}\right)}{2 \theta^{2}}-\frac{\operatorname{Var}_{0}\left(u_{1}^{1}\right)}{2 \theta^{2}}\right]+\lambda_{s}^{\xi} \cdot \xi,
\end{aligned}
$$

where

$$
\lambda_{s}^{\xi}=\frac{\lambda_{u_{1}^{1}}^{\xi}-\lambda_{u_{1}^{2}}^{\xi}}{\theta}=\frac{2(2 \lambda-1)}{\theta-\beta(\bar{s})\left(1+e^{\bar{s}}\right)}
$$

is the elasticity of the log-ratio of the Pareto weights with respect to the underlying shock.

When $\lambda>1 / 2$ and $\theta<0$, then $\lambda_{s}^{\xi}<0$. This means that when the supply of good $X$ is relatively scarce (i.e., $\xi<0$ ), agent 1 , whose preferences are relatively more tilted toward the consumption of this good, is compensated by means of a greater transfer of resources (i.e., $s$ increases). If $\lambda=1 / 2$, then the reallocation is null $\left(\lambda_{s}^{\xi}=0\right)$. This is a relevant case to consider because it corresponds to the situation in which the multiplegoods economy is equivalent to a one-good economy. As documented by Anderson (2005), in such an economy the distribution of resources is constant over time, unless preference heterogeneity is introduced. 
Conditional expectation of $s-\bar{s}$ We can characterize the drift in the log-ratio of the Pareto weights in equation (13) as follows:

$$
\begin{aligned}
{\left[\frac{\operatorname{Var}_{0}\left(u_{1}^{2}\right)}{2 \theta^{2}}-\frac{\operatorname{Var}_{0}\left(u_{1}^{1}\right)}{2 \theta^{2}}\right] } & =\frac{\sigma^{2}}{2 \theta^{2}}\left[\left(\lambda_{u_{1}^{2}}^{\xi}\right)^{2}-\left(\lambda_{u_{1}^{1}}^{\xi}\right)^{2}\right] \\
& =\underbrace{\frac{\sigma^{2} \beta(\bar{s})(2 \lambda-1)}{\theta-\beta(\bar{s})\left(1+e^{\bar{s}}\right)}}_{<0} \cdot \underbrace{\left(\theta^{-1} \lambda_{s}^{\xi}\right)}_{>0} \cdot\left(e^{\bar{s}}-1\right) .
\end{aligned}
$$

The value of the drift is pinned down by precautionary motives related to continuation utility variance. When the consumption share of agent 1 rises (i.e., $\bar{s}>0$ ), agent 1 wants to buy an increasing amount of insurance from agent 2 . In equilibrium, agent 1 accepts a reduction in her expected average share of resources (i.e., the drift in $(s-\bar{s})$ is negative) in exchange for a reduction in future utility variance. At the same time, agent 2 provides limited insurance at a higher price to agent 1 and expects to receive a greater future consumption share. ${ }^{2}$ In Appendix A in the Supplemental Material, we quantify this intuition further and show that the expected growth of the agent with the smaller consumption share increases with a larger degree of preference for one of the two goods $(\lambda)$, more fundamental risk $\left(\sigma^{2}\right)$, and stronger risk sensitivity $(\theta)$.

\subsection{Infinite-horizon model}

In this section, we prove that under symmetry a nondegenerate limiting distribution of Pareto weights exists. Equivalently, in the limit no agent receives a Pareto weight of zero with probability one. Furthermore, we characterize the adjustment of the Pareto weights as a function of the realization of the shocks, and the conditional expectation of the Pareto weights as a function of the current state of the economy.

An illustrative example We introduce a simple example used in the subsequent sections to better illustrate the properties of the model. Endowments can take on one of the following four equally likely pairs of realizations:

$$
\mathcal{N}=\{(X=103, Y=103),(X=103, Y=100),(X=100, Y=103),(X=100, Y=100)\} .
$$

We assume that the coefficient $\lambda_{1}=1-\lambda_{2}=0.97$ so that agent 1 enjoys a higher period utility when the supply of good $X$ is large and agent 2 is better off when good $Y$ is more abundant. The risk-sensitive parameter $\theta$ is set to $(1-\gamma)^{-1}$ where $\gamma=25$ in order to enhance the role of risk-sensitivity in this basic setup. We consider lower values of risk aversion in Sections 4 and 5. The qualitative implications of the model are the same as long as $\gamma>1$. For both agents, the subjective discount factor, $\delta$, is set to 0.96 to ensure fast convergence of our algorithm.

\footnotetext{
${ }^{2}$ We report the budget constraints associated to the decentralized economy in Appendix A in the Supplemental Material (available on the journal website, http://qeconomics.org/supp/457/code_and_data.zip).
} 
Ranking of Pareto weights We use the following proposition to characterize the ranking of Pareto weights as a function of the state of the economy.

Proposition 1. Let Assumptions 1-8 hold. Let the events $a, b \in \mathcal{N}$ be such that

$$
X^{\prime}(a) / Y^{\prime}(a)>X^{\prime}(b) / Y^{\prime}(b) .
$$

Then the ratio of Pareto weights is such that

$$
S^{\prime}(a, S) \leq S^{\prime}(b, S)
$$

If $X^{\prime}(a) / Y^{\prime}(a)=X^{\prime}(b) / Y^{\prime}(b)$, then $S^{\prime}(a, S)=S^{\prime}(b, S)$.

Proof. See Appendix B.1 in the Supplemental Material (available in a supplementary file on the journal website, http://qeconomics.org/supp/457/code_and_data.zip).

The interpretation of Proposition 1 is simple: whenever agent 1 receives a good shock to the endowment of the good that she likes the most, the social planner reduces her weight. This reallocation enables agents 1 and 2 to share part of the endowment risk of the economy, and it is consistent with what is shown in our two-period model, where we document that the elasticity $\lambda_{u_{1}^{1}}^{\xi}$ is negative. Furthermore, if the two goods are in identical supply, the optimal choice of Pareto weights is independent of the supply level.

Figure 1 documents this ranking by showing the optimal policies associated with our illustrative example. First, notice that the optimal policy is identical in the two states of equal supply of the two goods (see the top-left and the bottom-right panel). Second, notice that next-period's Pareto weight attached to agent 1 is lower when the supply of good $X$ is relatively more abundant (top-right panel) than it is when the supply of good $X$ is relatively more scarce (lower-left panel).

The distribution of the Pareto weights: Ergodicity and mean reversion We document that in the limit no agent receives a Pareto weight of zero with probability one. Furthermore, it is possible to demonstrate that in our economy the dynamics of Pareto weights are characterized by mean reversion. This means that when the Pareto weight of any agent is small (large), it is expected to increase (decrease) going forward. The following two propositions formalize these statements.

Proposition 2. Let Assumptions 1-8 hold. The stochastic processes $\mu_{1}$ and $\mu_{2}$ cannot converge to either 0 or 1 almost surely.

Proof. See Appendix B.2 in the Supplemental Material (available in a supplementary file on the journal website, http://qeconomics.org/supp/457/code_and_data.zip).

Proposition 3. Let Assumptions 1-8 hold. The expectation of the next-period's Pareto weight on agent 1 conditional on the current Pareto weight is such that (i) $E\left[\mu_{1}^{\prime} \mid S\right]=\mu_{1}$, if $\mu_{1}=\frac{1}{2}$; (ii) $E\left[\mu_{1}^{\prime} \mid S\right]<\mu_{1}, \forall \mu_{1} \in\left(\frac{1}{2}, 1\right)$; and (iii) $E\left[\mu_{1}^{\prime} \mid S\right]>\mu_{1}, \forall \mu_{1} \in\left(0, \frac{1}{2}\right)$. 

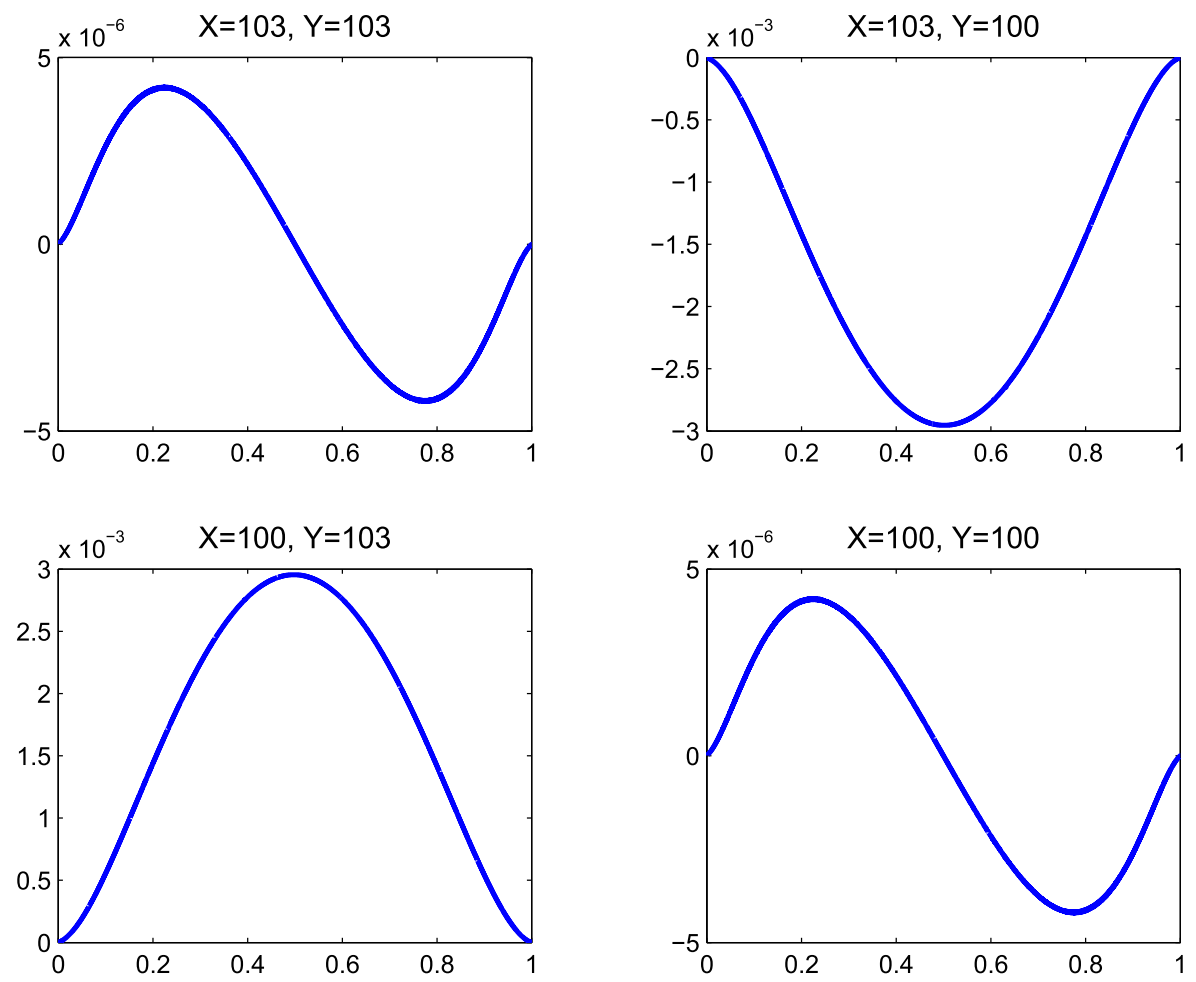

Figure 1. Phase diagrams of Pareto weights. Each panel refers to a different realization of the endowment of the two goods at time $t+1, z_{t+1}=\left[X_{t+1} Y_{t+1}\right]$. On the vertical axis, we depict the difference between the future Pareto weight for agent $1, \mu_{1, t+1}=f_{\mu_{1}}\left(z_{t+1}, \mu_{1, t}\right)$, and its current value, $\mu_{1, t}$. On the horizontal axis, we have $\mu_{1, t}$.

Proof. See Appendix B.3 in the Supplemental Material (available in a supplementary file on the journal website, http://qeconomics.org/supp/457/code_and_data.zip).

The content of Propositions 2 and 3 is depicted in Figure 2. The left panel of Figure 2 shows that the invariant distribution of Pareto weights does not display any mass in the limiting cases of $\mu_{1}=\{0,1\}$. This means that in the long run both agents "survive," that is, they both consume a nonzero share of the aggregate resources.

The right panel of Figure 2 shows that the conditional change in the Pareto weight of each agent is positive when the Pareto weight is small, and negative when the Pareto weight is large. Equivalently, the dynamics of the Pareto weight feature mean reversion, which is due to endogenous asymmetries in precautionary saving motives. Taken together, Propositions 2 and 3 ensure the existence of a well-defined invariant distribution of Pareto weights.

As in our two-period economy, the substitution effect generated by the reallocation channel is size dependent due to the nonlinearity of the aggregator of the two goods. An agent with a large share of consumption benefits the least from the substitution effect and is willing to buy very expensive insurance from the other agent in order to reduce 

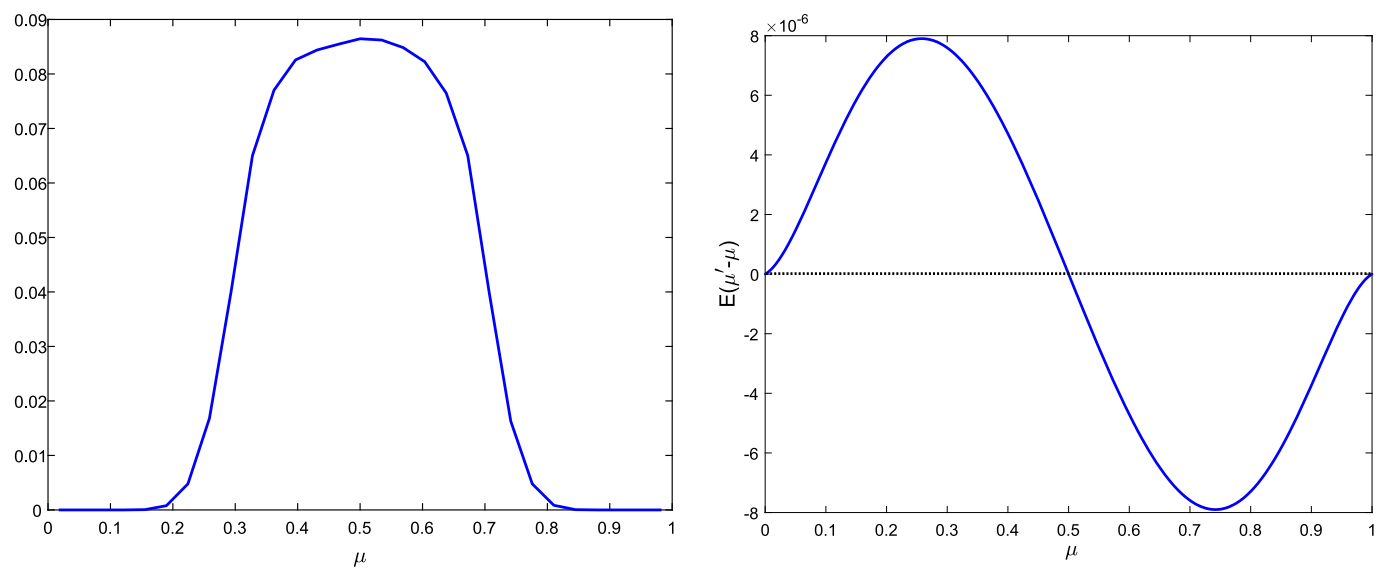

Figure 2. Survivorship. The left panel reports the invariant distribution of the Pareto weight of agent $1\left(\mu_{1}\right)$. The right panel shows the conditional expectation of the Pareto weight increment for agent $1, E\left[\mu_{1}^{\prime}-\mu_{1} \mid \mu_{1}\right]$, as a function of the current $\mu_{1}$.

the conditional variance of her continuation utility. As a result, the agent with a small consumption share is expected to receive a positive transfer of resources going forward, and her consumption share is expected to become larger.

\section{Comparison of approximations}

In this section, we investigate the ability of both first- and higher-order approximations to capture the short- and long-run characteristics of the model. In order to use common perturbation techniques, we assume that endowments are jointly log-normally distributed, with the following means and covariance matrix:

$$
\left(\begin{array}{l}
\log X \\
\log Y
\end{array}\right) \sim N\left(\left[\begin{array}{l}
4.62 \\
4.62
\end{array}\right],\left[\begin{array}{cc}
0.025^{2} & 0.3 \cdot 0.025^{2} \\
0.3 \cdot 0.025^{2} & 0.025^{2}
\end{array}\right]\right) .
$$

This calibration captures the degree of correlation of output (Colacito and Croce (2013)).

In what follows, we show that the results with Gaussian shocks are similar to those obtained with a finite discretized joint normal distribution. This constitutes a generalized setup relative to our earlier sections, which will prove important in allowing us to numerically analyze several interesting extensions of our benchmark model in Section 5. Specifically, we discretize the distribution of the exogenous endowment shocks on a $21 \times 21$ grid of equally spaced nodes on the range

$$
[\exp \{4.62-5 \times 0.025\}, \exp \{4.62+5 \times 0.025\}] .
$$

We set $\delta=0.96, \lambda_{1}=0.97$, and $\gamma=15$. This value of risk aversion is in line with those typically employed in the equity premium puzzle literature (see, among others, Tallarini (2000)) and can be lower than that used in Section 3.2 because we adopt a richer set of exogenous states. 


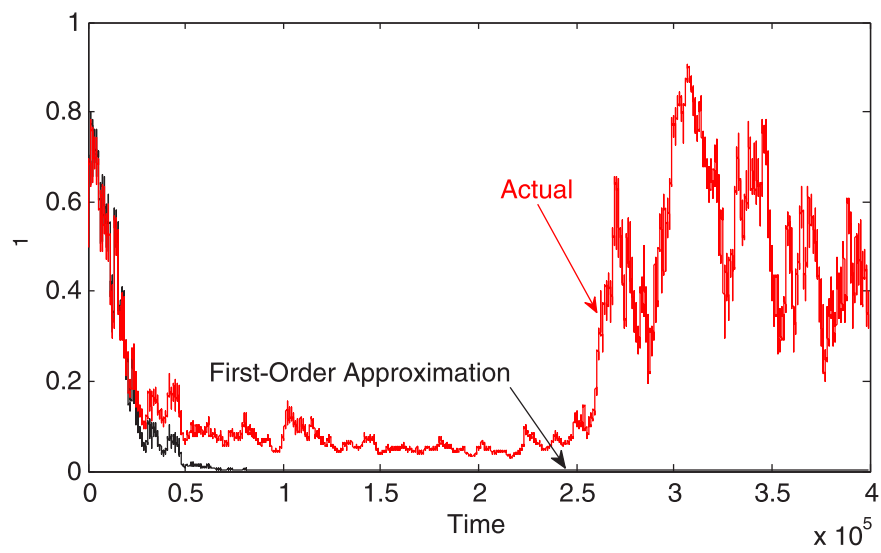

Figure 3. Comparison of the actual dynamics of Pareto weights and that obtained through a first-order Taylor expansion. For the same sequence of shocks, the black line shows the actual path of the agent 1 Pareto weight, $\mu_{1}$, while the red line shows the path obtained using a first-order approximation about the unconditional mean of $\mu_{1}$, that is, $\bar{\mu}_{1}=1 / 2$.

\section{The curse of the linear approximation}

A first-order Taylor approximation about the unconditional mean of the ratio of Pareto weights fails to reproduce at least two crucial aspects of the economy. First, it provides a highly inaccurate description of the period-by-period dynamics of the model. Second, and most importantly, it does not capture the mean-reverting property of the model. This results in the possibility that one of the two agents eventually dies and is assigned a steady-state Pareto weight of zero.

In order to show these two facts, we proceed as follows. First, we solve the model numerically by value-function iteration (see Appendix C in the Supplemental Material, available in a supplementary file on the journal website, http://qeconomics.org/supp/ 457/code_and_data.zip) and obtain what we denote as the "actual" solution. Second, we compare the "actual" dynamics of Pareto weights with the dynamics computed using a first-order Taylor expansion about $\mu_{1}=\mu_{2}=0.5$. Figure 3 reports this comparison for a simulation of 400,000 periods.

For the first part of the simulation, the Pareto weights are in the relatively small neighborhood of 0.5. In this region, the first-order Taylor expansion does a good job of approximating the actual dynamics of the economy. The approximation, however, starts deteriorating significantly as the economy departs from $\mu_{1}=0.5$. On this history, according to the first-order Taylor expansion, the Pareto weight of agent 1 should level off at zero, even though this is in sharp contrast to the actual dynamics of the model and the survivorship results explained in the previous sections. As a consequence, the long-run implications of the first-order Taylor expansion are unreliable.

In this clear-cut example, what may at first look like a small error results in an irreversible misrepresentation of the actual dynamics of the economy and its long-run moments. In this economy, higher-order approximations are needed not only to provide a more accurate description of the period-by-period dynamics, but also to preserve the existence of a well-defined ergodic distribution. 

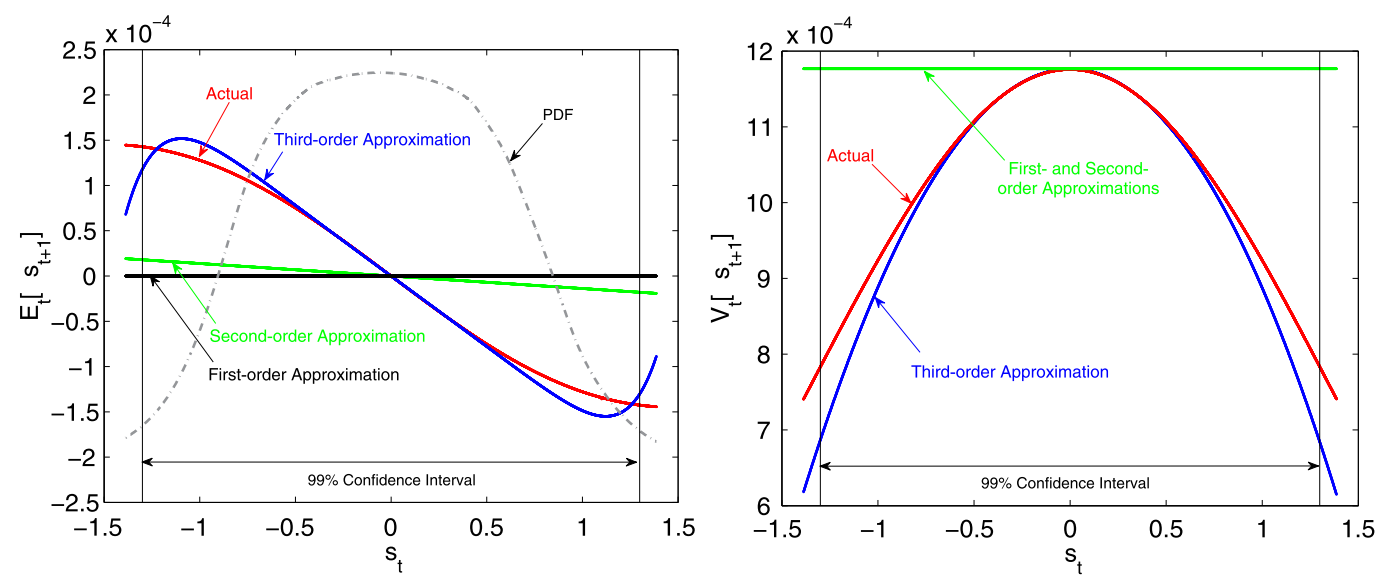

Figure 4. Comparison of first and second conditional moments across several solution methods. We denote the log of the ratio of the Pareto weights by $s_{t} \equiv \log \left(S_{t}\right)$ and its growth by $\Delta s_{t+1}=s_{t+1}-s_{t}$, respectively. The left panel reports the conditional mean $E\left[\Delta s_{t+1} \mid s_{t}\right]$ as a function of $s_{t}$. The right panel reports the conditional variance of $\Delta s_{t+1}$ with respect to $s_{t}$. In each panel, the curve label "Actual" refers to the solution obtained through value-function iterations. The other three curves are based on Taylor approximations of higher order. In the left panel, the probability density function (PDF) is computed using the actual policy.

\section{The case for higher-order approximations}

The linear approximation does not accurately describe the dynamics of the Pareto weights because it impels a first-order integrated process. This is clearly depicted in the left panel of Figure 4, in which we compare the expected growth of the ratio of Pareto weights as a function of the current ratio across different solution methods. The flat line for the first-order approximation suggests that the conditional expected change of the ratio of Pareto weights is identically zero, implying the lack of any kind of mean reversion.

The second-order approximation does capture some of the mean reversion, although not enough to be comparable to the actual solution of the model. Furthermore, by looking at the right panel of Figure 4 we notice that the second-order approximation does not feature any time variation in the conditional variance of the ratio of Pareto weights. This is in stark contrast to the actual dynamics of the second moments of the actual solution. Equivalently, the second-order approximation completely misses the time variation in the last term of equation (2), that is, the key determinant of the risk-sharing motive of our agents.

In order to capture time-varying volatilities of both consumption and continuation utilities, we implement a third-order approximation. In contrast to the second-order perturbation, the third-order approximation provides an extremely accurate representation of the dynamics of both the first and second conditional moments. This is certainly the case in a 99\% confidence interval of the actual long-run distribution of the ratio of Pareto weights. As expected, the quality of the approximation deteriorates toward the tails of the distribution. 
To summarize, this class of models produces rich dynamics for both the first and second conditional moments of the Pareto weights and, therefore, consumption shares across agents. To appropriately capture these dynamics, an approximation of at least the third order is required. In the next section, we use third-order approximations to study more general settings.

\section{More General ENVIRONMENTS}

In this section, we generalize our setting in two respects. First of all, we consider preferences defined as in Epstein and Zin (1989),

$$
U_{i, t}=\left[(1-\delta) \cdot\left(C_{i, t}\right)^{1-1 / \psi}+\delta E_{t}\left[\left(U_{i, t+1}\right)^{1-\gamma}\right]^{\frac{1-1 / \psi}{1-\gamma}}\right]^{\frac{1}{1-1 / \psi}}, \quad \forall i \in\{1,2\},
$$

where $\psi$ denotes the IES and $\gamma$ represents RRA. Second, we consider the following endowment process that allows persistence:

$$
\begin{aligned}
& \log X_{t}=\mu+\rho \log X_{t-1}-\tau\left[\log X_{t-1}-\log Y_{t-1}\right]+\varepsilon_{t}^{X}, \\
& \log Y_{t}=\mu+\rho \log Y_{t-1}+\tau\left[\log X_{t-1}-\log Y_{t-1}\right]+\varepsilon_{t}^{Y}, \\
& {\left[\begin{array}{c}
\varepsilon_{t}^{X} \\
\varepsilon_{t}^{Y}
\end{array}\right] \sim \operatorname{iidN}\left(\left[\begin{array}{l}
0 \\
0
\end{array}\right],\left[\begin{array}{cc}
\sigma^{X 2} & \rho_{X, Y} \sigma^{X} \sigma^{Y} \\
\rho_{X, Y} \sigma^{X} \sigma^{Y} & \sigma^{Y 2}
\end{array}\right]\right),}
\end{aligned}
$$

where $\rho \in[0,1]$ and $\tau \in(0,1)$ determine the extent of cointegration when $\rho=1$. Cointegration is required to have a well-defined ergodic distribution of the relative supply of the two goods, but it plays a minor quantitative role in our analysis as we set it to a very small number.

Solving the planner's problem with global methods and multiple exogenous state variables goes beyond the scope of this manuscript. The reason is that properly capturing the mean reversion of the pseudo-Pareto weights requires a very thin grid, and it exposes us to the curse of dimensionality even with one extra state. Hence in this section we explore the generality of our results through simulations based on a third-order perturbation of our dynamic model, which we detail in Appendix D in the Supplemental Material (available in a supplementary file on the journal website, http://qeconomics. org/supp/457/code_and_data.zip).

Reference calibration Our reference calibration features $\mu=2 \%, \rho=0.90, \sigma^{X}=$ $\sigma^{Y}=1.87 \%, \rho_{X, Y}=0.35, \tau=5.0 \mathrm{E}-04, \gamma=5, \psi=1, \delta=0.96$, and $\lambda_{1}=\lambda_{2}=0.97$. The parameters for the endowment processes are set in the spirit of Colacito and Croce (2013). In what follows, we first consider different endowment processes and different levels of the IES and RRA while preserving symmetry across agents and goods. We then explore the implications for a small degree of heterogeneity in preference for the two goods $\left(\lambda_{i}\right)$ and in fundamental volatility across goods $\left(\sigma^{X}\right.$ and $\left.\sigma^{Y}\right)$.

\subsection{Symmetric environments}

The role of persistence We vary the persistence of our endowment shocks from zero to one. When $\rho=0$, we have i.i.d. level shocks, as in the previous section. When $\rho=1$, level 

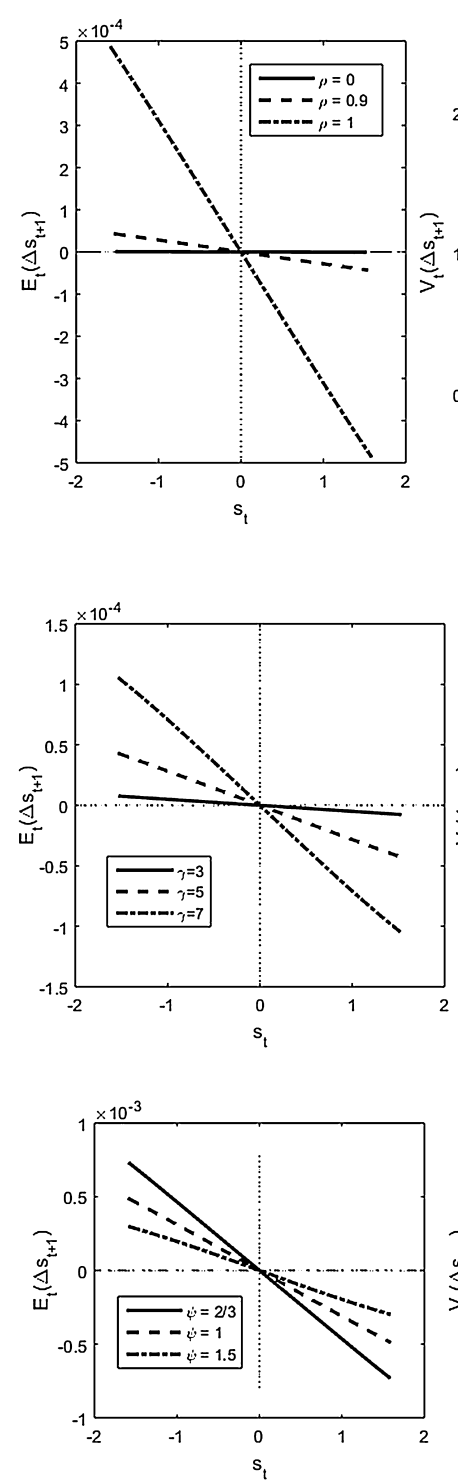

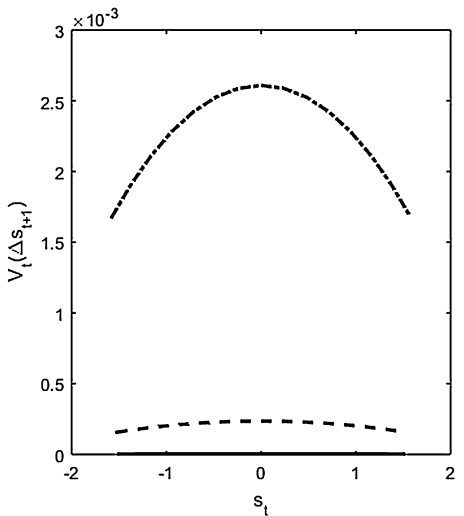

(a) Persistence

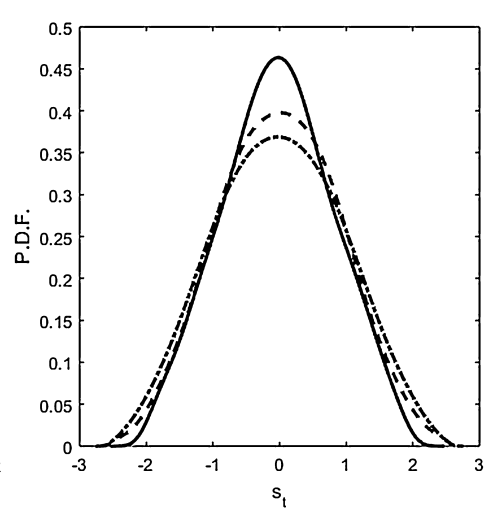

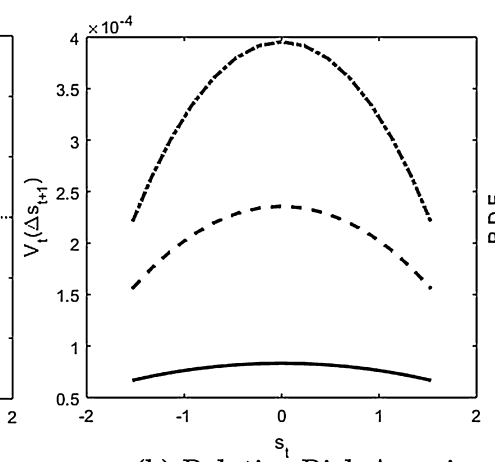

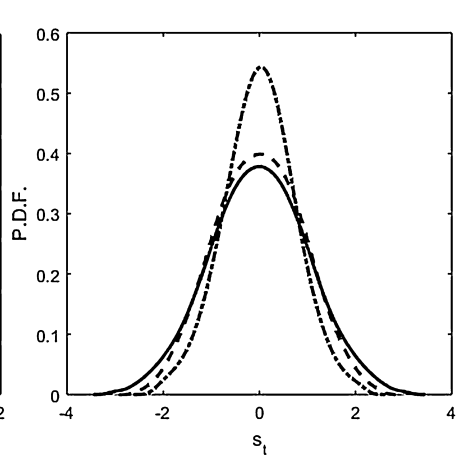

(b) Relative Risk Aversion
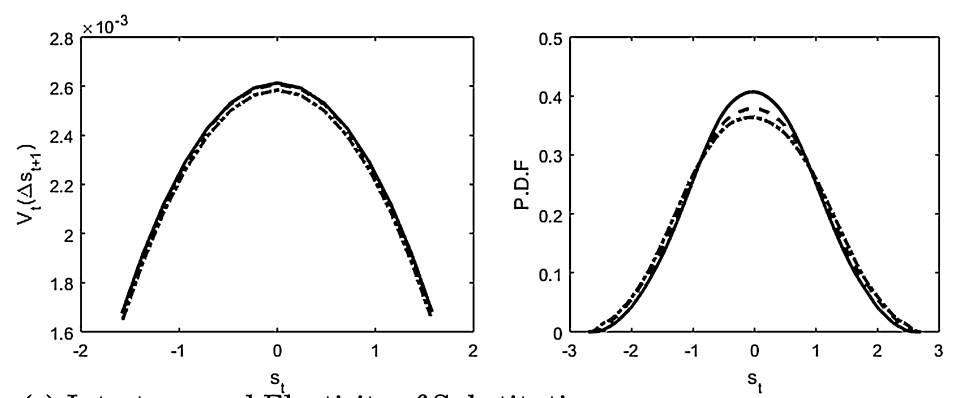

(c) Intertemporal Elasticity of Substitution

Figure 5. The role of persistence and preferences. Our model features Epstein and Zin (1989) preferences as specified in equation (14) and the endowments reported in equation (15). Our reference calibration is detailed in Section 5. We vary one parameter at a time for both countries, leaving the other parameters unchanged.

shocks are permanent. We depict key features of the distribution of the log-ratio of the Pareto weights, $s_{t}$, in Figure 5 and simulated moments in Table 1.

We make several observations. First, as we increase $\rho$, the endowment shocks become more long-lasting and volatile. As a result, the endogenous process $s_{t}$ becomes more volatile, as documented by its fatter tails (rightmost plot of Figure 5, panel A) and the higher conditional volatility of $\Delta s_{t}$ (center plot of Figure 5). 
TABLE 1. The role of persistence and preferences.

\begin{tabular}{|c|c|c|c|c|c|c|c|}
\hline \multirow{2}{*}{$\begin{array}{l}\text { Parameter } \\
\text { Value }\end{array}$} & \multicolumn{2}{|c|}{ Moments of SWC } & \multicolumn{4}{|c|}{ Percentiles of Approx. Errors (\%) } & \multirow{2}{*}{$\begin{array}{l}\text { Cumul. } \\
\text { ERR }(\%\end{array}$} \\
\hline & Mean & Std. Dev. & 50 & 75 & 90 & 95 & \\
\hline \multicolumn{8}{|c|}{ The role of persistence $(\rho)$} \\
\hline 0 & 0.5 & 0.18 & $2.0 \mathrm{E}-14$ & $4.0 \mathrm{E}-14$ & $7.0 \mathrm{E}-14$ & $9.0 \mathrm{E}-14$ & $8.0 \mathrm{E}-01$ \\
\hline 0.9 & 0.5 & 0.19 & $8.0 \mathrm{E}-15$ & $2.0 \mathrm{E}-14$ & $2.0 \mathrm{E}-14$ & $4.0 \mathrm{E}-14$ & $7.0 \mathrm{E}-04$ \\
\hline 1 & 0.5 & 0.2 & $2.0 \mathrm{E}-14$ & $4.0 \mathrm{E}-14$ & $6.0 \mathrm{E}-14$ & $7.0 \mathrm{E}-14$ & $5.0 \mathrm{E}-05$ \\
\hline \multicolumn{8}{|c|}{ The role of RRA $(\gamma)$} \\
\hline 3 & 0.5 & 0.21 & $2.0 \mathrm{E}-14$ & $3.0 \mathrm{E}-14$ & $6.0 \mathrm{E}-14$ & $9.0 \mathrm{E}-14$ & $1.0 \mathrm{E}-02$ \\
\hline 5 & 0.5 & 0.2 & $8.0 \mathrm{E}-15$ & $2.0 \mathrm{E}-14$ & $2.0 \mathrm{E}-14$ & $4.0 \mathrm{E}-14$ & $7.0 \mathrm{E}-04$ \\
\hline 7 & 0.5 & 0.16 & $3.0 \mathrm{E}-14$ & $6.0 \mathrm{E}-14$ & $8.0 \mathrm{E}-14$ & $9.0 \mathrm{E}-14$ & $5.0 \mathrm{E}-04$ \\
\hline \multicolumn{8}{|c|}{ The role of IES $(\psi)$} \\
\hline $2 / 3$ & 0.5 & 0.19 & $4.0 \mathrm{E}-11$ & $4.0 \mathrm{E}-11$ & $4.0 \mathrm{E}-14$ & $4.0 \mathrm{E}-14$ & $1.0 \mathrm{E}-01$ \\
\hline 1 & 0.5 & 0.2 & $2.0 \mathrm{E}-14$ & $4.0 \mathrm{E}-14$ & $5.0 \mathrm{E}-14$ & $7.0 \mathrm{E}-14$ & $5.0 \mathrm{E}-05$ \\
\hline 1.5 & 0.5 & 0.21 & $4.0 \mathrm{E}-14$ & $9.0 \mathrm{E}-14$ & $2.0 \mathrm{E}-13$ & $2.0 \mathrm{E}-13$ & $5.0 \mathrm{E}-05$ \\
\hline
\end{tabular}

Note: Our model features Epstein and Zin (1989) preferences as specified in equation (14) and the endowments reported in equation (15). Our reference calibration is detailed in Section 5 . We vary one parameter at a time, leaving the others unchanged. Both the approximation errors and the cumulative approximation errors (Cumul. ERR) are defined in Appendix D and are multiplied by 100 . All numbers are based on repetitions of long-sample simulations (at least 2 million periods) with different starting points for log ratio of the Pareto weights $\left(s_{t}\right)$.

The unconditional volatility of $s_{t}$, however, increases just slightly, as documented in Table 1. This is because as shocks become more long lasting the precautionary motives of these agents become more pronounced and more sensitive to size. As the exogenous endowment persistence $\left(\rho\right.$ ) increases, the endogenous persistence of $s_{t}$ declines, as captured by the more negative slope of $E_{t}\left[\Delta s_{t+1}\right]$ with respect to $s_{t}$ (leftmost plot of Figure 5, panel A).

Second, given the lower persistence of $s_{t}$, our cumulative error measure declines to very small numbers with higher values of $\rho$. This result is reassuring because in many realistic applications the endowment shocks are calibrated to be very persistent.

The role of preferences When we vary the subjective discount factor, we do not find significant changes in the dynamics of the log-ratio of the Pareto weights. For this reason, we focus only on the role of risk aversion and IES. Increasing risk aversion amplifies the sensitivity of continuation utility to shocks, and hence it makes the redistribution channel stronger. This intuition is confirmed in panel (b) of Figure 5, where we show that the conditional variation of $\Delta s_{t+1}$ increases with higher values of $\gamma$ and, at the same time, the mean reversion of the Pareto weights speeds up.

Since the endogenous change in mean reversion dominates quantitatively, as $\gamma$ increases more mass is concentrated in the center of the probability distribution function of $s_{t}$, implying that the unconditional volatility of this process declines. Together, the faster speed of mean reversion and the lower unconditional volatility of $s_{t}$ imply lower levels of approximation errors.

We conclude this analysis by examining the case in which we vary the IES. The effect of this parameter on the conditional volatility of the ratio of Pareto weights is almost 
negligible (center plot of panel (c), Figure 5). The impact on the endogenous persistence of $s_{t}$ is a bit more pronounced, but still moderate compared to the case in which we change risk aversion. Qualitatively, agents with a higher IES are more willing to accept fluctuations of consumption over time and hence are more willing to accept very long-lasting reallocations, that is, slower mean reversion in $s_{t}$ (leftmost plot in panel (c), Figure 5). Most importantly, we note that in the long-run risk literature the IES is set to values larger than or equal to one. For these values, the approximation errors are small, meaning that when the curvature of the utility function with respect to intertemporal aggregation is moderate $(\psi \geq 1)$, the quality of our approximation is good.

\subsection{Asymmetric environments}

Asymmetry preferences for the two goods We lower the degree of preference for good $Y$ of agent $2, \lambda_{2}$, from 0.97 to 0.90 , thus increasing the ability of agent 2 to smooth fluctuations in her consumption bundle by trading the two goods. All other parameters are unchanged. We depict key features of the distribution of the log-ratio of the Pareto weights, $s_{t}$, in Figure 6 and simulated moments in Table 2.

Since agent 2 faces less consumption uncertainty than agent 1 , her demand of insurance is moderate compared to that of agent 1 . As a result, the average share of resources allocated to agent 2 increases and the curves depicted in the top-left panel of Figure 6 shift to the left. In the Appendix in the Supplementary Material, we show that this intuition is also present in the simple two-period model (see Figure AF-1).

We note that the distribution of the log-ratio of the Pareto weights does not shift to the left in a parallel way. As documented in the top portion of Table 2, under the optimal risk-sharing scheme, agent 1 accepts a lower average level of resources in exchange for both a reduction in future utility uncertainty and positive skewness of its share of world consumption. That is, agent 1 benefits from a sizeable positive redistribution of resources along histories with a severe downside of the relative supply of good $X$.

Rabitsch, Stepanchuk, and Tsyrennikov (2015) point out that a global approximation is required when countries are subject to asymmetric constraints, such as a borrowing limit, and when their wealth distribution is nonstationary. Since we have a frictionless model with complete markets and a well-defined ergodic distribution of wealth, a perturbation approach provides a good approximation of the equilibrium. Consistent with the findings in Rabitsch, Stepanchuk, and Tsyrennikov (2015), our cumulative errors increase as we make the two agents more asymmetric, but our errors remain as low as $2.2 \%$.

CRRA and heterogeneous degree of preference for the two goods It is useful to study this asymmetric scenario under time-additive CRRA preferences. We choose the intermediate case $\lambda_{1}=0.97, \lambda_{2}=0.95$, and set $\psi^{-1}=\gamma=5 .{ }^{3}$ We fix the initial ratio of the Pareto weights to a value that delivers an average SWC of 0.47 , as in the case with recursive preferences. Panel (b) of Figure 6 and Table 2 confirm that under recursive preferences, the

\footnotetext{
${ }^{3}$ Note that since $\gamma>1$, the ratio of Pareto weights is no longer constant over time despite the adoption of time additivity of preferences (Cole and Obstfeld (1991)). The implied variation in $s_{t}$, however, is very limited as highlighted in panel (b) of Figure 6.
} 

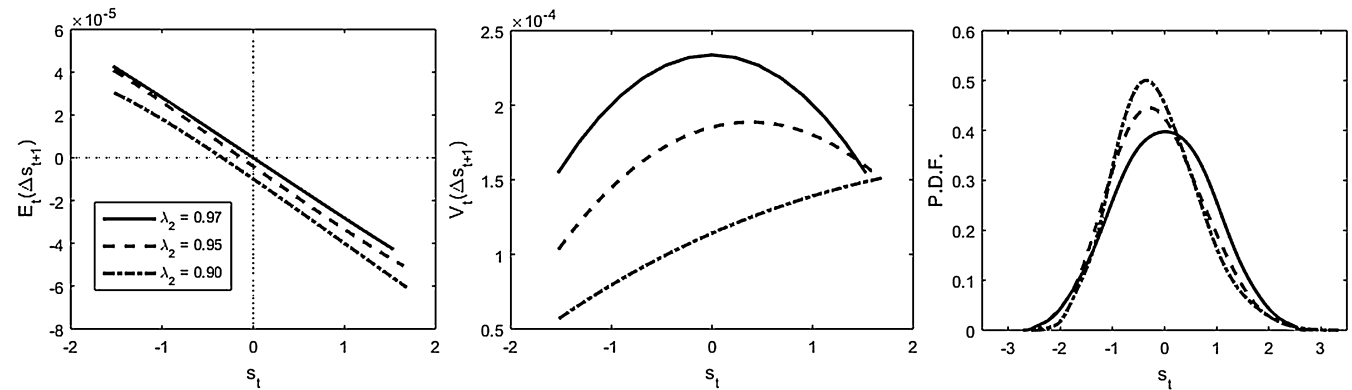

(a) Asymmetries in the degree of preference for the two goods
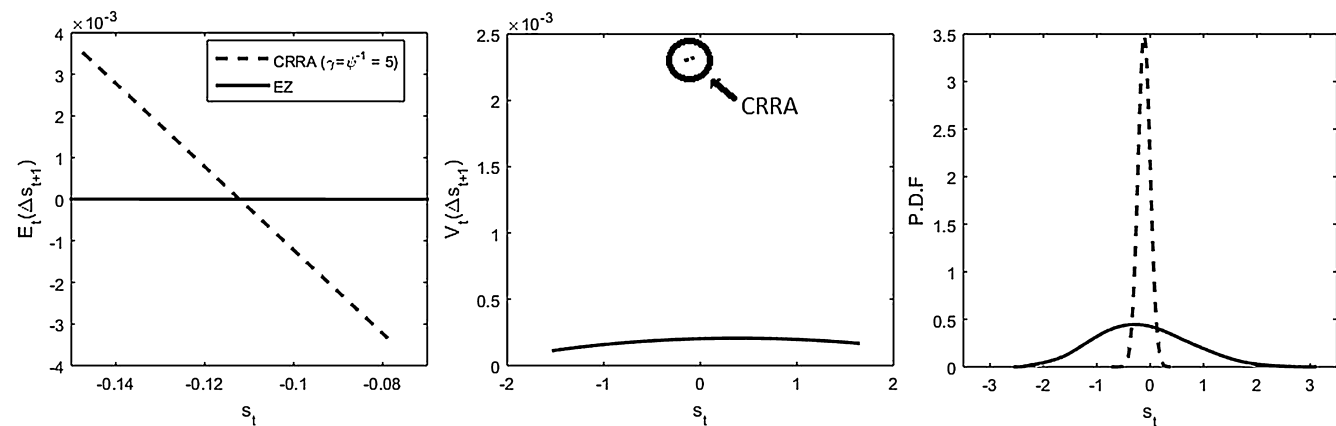

(b) Asymmetries in the degree of preference for the two goods - CRRA versus EZ
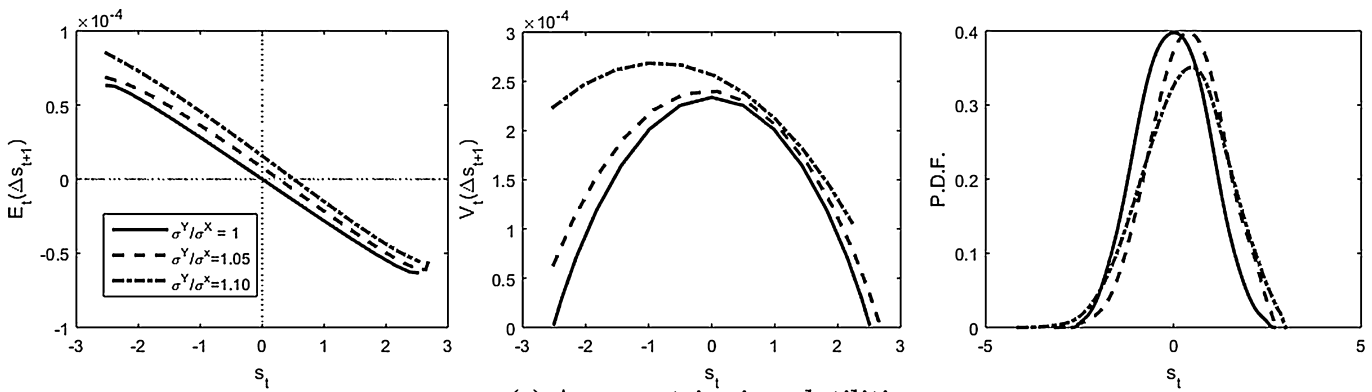

(c) Asymmetries in volatilities

FiguRE 6. Asymmetric calibrations. Our model features Epstein and Zin (1989) preferences as specified in equation (14) and the endowments reported in equation (15). Our reference calibration is detailed in Section 5. We vary one parameter at a time, leaving the others unchanged.

reallocation channel is very pronounced and long-lasting, and it prescribes a significant amount of positive skewness for agent 1 , as she is facing more consumption risk because of a higher degree of preference for good $X$.

Heterogeneous volatility In many applications, the properties of the goods traded are asymmetric. In international finance, for example, different countries may be subject to productivity shocks with different volatilities. In the presence of the same degree of preference for the two goods, the agent which prefers the more volatile good faces more consumption, and hence future utility uncertainty. As suggested by our two-period model 
TABle 2. Asymmetric cases.

\begin{tabular}{lcccc}
\hline \multirow{2}{*}{$\begin{array}{l}\text { Parameter } \\
\text { Value }\end{array}$} & \multicolumn{3}{c}{ Moments of SWC } & Cumul \\
\cline { 2 - 5 } ERR $(\%)$ & Skew & \\
\hline Agent 2 Bias $\left(\lambda_{2}\right)-$ EZ Case & & Std & 0 & $7.0 \mathrm{E}-04$ \\
0.97 & 0.5 & 0.19 & 0.22 & $2.6 \mathrm{E}-03$ \\
0.95 & 0.47 & 0.18 & 0.33 & \\
0.90 & 0.46 & 0.17 & & $5.0 \mathrm{E}-06$ \\
Agent 2 Bias $\left(\lambda_{2}\right)-$ CRRA Case $\left(\gamma=\psi^{-1}=5\right)$ & & 0.03 & \\
0.95 & 0.47 & 0.02 & & $7.0 \mathrm{E}-04$ \\
Y-Good Volatility $\left(\sigma^{Y}\right)$ & & & 0 & $9.0 \mathrm{E}-04$ \\
$1.00 \cdot \sigma^{X}$ & 0.5 & 0.19 & -0.27 & $1.3 \mathrm{E}-01$ \\
$1.05 \cdot \sigma^{X}$ & 0.58 & 0.19 & -0.27 & \\
$1.10 \cdot \sigma^{X}$ & 0.56 & 0.22 & & \\
\hline
\end{tabular}

Note: Our model features Epstein and Zin (1989) preferences as specified in equation (14) and the endowments reported in equation (15). Our reference calibration is detailed in Section 5 . We vary one parameter at the time, leaving the others unchanged. The cumulative approximation errors (Cumul ERR) are defined in Appendix D and are multiplied by 100 . All numbers are based on repetitions of long sample simulations (at least 2 million periods) with different starting points for log ratio of the Pareto weights $\left(s_{t}\right)$.

(see Figure AF-1), this agent should be willing to accept a low average SWC in exchange for insurance. Panel (c) of Figure 6 and Table 2 confirm this finding as we increase $\sigma^{Y}$.

In this case, the average share of resources of agent 1 increases. The volatility of the SWC increases as well, as a direct result of the higher standard deviation of good $Y$. Agent 1 is willing to insure agent 2 against downside risk, and agent 1 accepts negative skewness in her own share of resources. Across all cases, the associated cumulative approximation errors are smaller than $0.14 \%$.

Heterogenous RRA A well-known result with multiple agents with risk-sensitive preferences in a one-good economy with growth is that only the agent with the lowest risk aversion remains wealthy in the long run, ceteris paribus (see Anderson (2005)). We confirm this finding in our setting by depicting in Figure 7 the expected growth rate of the log-ratio of Pareto weights for the case $\lambda_{1}=\lambda_{2}$ and $\gamma_{2}>\gamma_{1}$. Since the expected growth is positive for all values of $s_{t}$, all resources are allocated to agent 1 in the limit.

Our risk-sharing mechanism, however, suggests that as we make the degree of preference for the two goods more asymmetric, size matters progressively more for the intensity of the reallocation channel. Hence as the high-risk-aversion agent receives a smaller share of world resources, her willingness to buy further insurance should decline, which in turn results in survivorship. Our simulations confirm this intuition and suggest that there exist regions of the parameter space in which survivorship is also possible with asymmetric risk aversion, provided that multiple parameters are simultaneously adjusted, in the spirit of Anderson (2005).

\section{Concluding Remarks}

We have characterized the solution of a planner's problem with multiple agents, multiple goods, and recursive preferences. The introduction of multiple goods substantially 


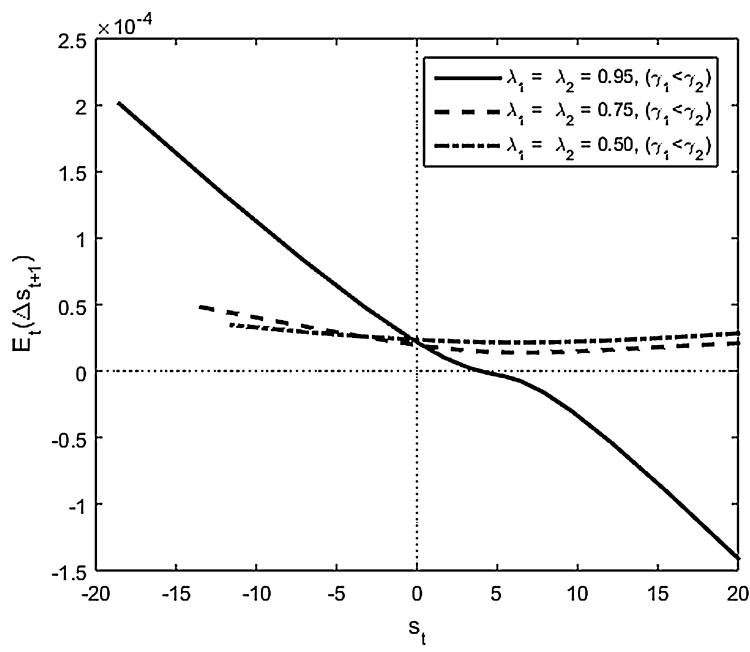

Figure 7. Asymmetric risk aversion. Our model features Epstein and Zin (1989) preferences as specified in equation (14) and the endowments reported in equation (15). Our reference calibration is detailed in Section 5. We set $\gamma_{2}=7$ and $\gamma_{1}=5$ and vary simultaneously $\lambda_{1}$ and $\lambda_{2}$.

changes the dynamics of Pareto optimal allocations. Future research should extend our theoretical results to continuous time and continuous shocks. Asset pricing implications may be particularly appealing, due to the ability of this class of models to endogenously produce time-varying second moments without requiring market frictions. Since the main features of the risk-sharing scheme can be accurately captured through a thirdorder approximation, this class of models could be easily extended to an international business cycle setting as well.

\section{Appendix A: Two-Period MOdel}

\section{Environment}

In this section, we present a simplified two-period version of our model in order to provide intuition on the reallocation motives induced by recursive preferences. Specifically, at time $t=1$ agents receive news $\xi$ about their time- 1 endowment of goods. At time $t=0$, that is, before the arrival of the shock, agents $i \in\{1,2\}$ exchange a complete set of $\xi$-contingent securities to maximize their time- 0 utility, given their initial wealth (reflected in their time-0 Pareto weights).

\section{Utility and technology}

In what follows, we take advantage of lognormality wherever possible. Up to a log linearization of the allocation shares, this modeling strategy enables us to obtain a simple closed-form solution. In this spirit, we start by assuming that agents have an IES equal 
to 1 , that is, their preferences can be expressed as follows:

$$
u_{0}^{i}= \begin{cases}\delta \theta_{i} \log E_{0}\left[\exp \left\{\frac{u_{1}^{i}}{\theta_{i}}\right\}\right], & 0>\theta_{i}>-\infty \\ \delta E_{0}\left[u_{1}^{i}\right], & \theta_{i} \rightarrow-\infty\end{cases}
$$

where

$$
u_{1}^{i}=\log \left(C_{1}^{i}\right)
$$

and

$$
C_{1}^{1}=\left(X_{1}^{1}\right)^{\lambda_{1}}\left(Y_{1}^{1}\right)^{\left(1-\lambda_{1}\right)}, \quad C_{1}^{2}=\left(X_{1}^{2}\right)^{\left(1-\lambda_{2}\right)}\left(Y_{1}^{2}\right)^{\lambda_{2}} .
$$

The resource constraints are specified as follows:

$$
X_{1}^{1}+X_{1}^{2}=e^{\xi}, \quad Y_{1}^{1}+Y_{1}^{2}=e^{-l \xi}, \quad \xi \sim N\left(0, \sigma^{2}\right),
$$

where the parameter $l$ determines whether agent 2 is more $(l>1)$ or less $(0<l<1)$ exposed to the shock $\xi$ than agent 1 . We assume that $\xi$ affects both goods to preserve symmetry in our equations. In what follows, we show that the results are driven only by the relative supply of the goods $(1+l) \xi$.

\section{Pareto problem}

Under complete markets, the allocation can be recovered by solving the following Pareto problem:

$$
\max _{\left\{X_{1}^{1}, X_{1}^{2}, Y_{1}^{1}, Y_{1}^{2}\right\}} \mu_{0} \cdot u_{0}^{1}+\left(1-\mu_{0}\right) \cdot u_{0}^{2}
$$

subject to the constraints specified in (A.2)-(A.3). Let $S_{0} \equiv \mu_{0} /\left(1-\mu_{0}\right)$; after simplifying common coefficients, the optimality condition for the allocation of good $X_{1}$ is

$$
S_{1}(\xi) \frac{\partial \log C_{1}^{1}}{\partial X_{1}}=\frac{\partial \log C_{1}^{2}}{\partial X_{1}}
$$

with

$$
S_{1}(\xi)= \begin{cases}S_{0}, & \theta_{i} \rightarrow-\infty, \\ S_{0} \cdot \frac{\exp \left\{\frac{u_{1}^{1}}{\theta_{1}}\right\}}{E_{0}\left[\exp \left\{\frac{u_{1}^{1}}{\theta_{1}}\right\}\right]} / \frac{\exp \left\{\frac{u_{1}^{2}}{\theta_{2}}\right\}}{E_{0}\left[\exp \left\{\frac{u_{1}^{2}}{\theta_{2}}\right\}\right]}, & 0>\theta_{i}>-\infty .\end{cases}
$$

Equation (A.4) establishes that the optimal allocation can be found as in a regular static problem, for a given value of $S_{1}$. Equation (A.5) pins down $S_{1}$ and yields two important results. First, in the time-additive case, the share of resources is time invariant, that is, it is not affected by the actual realization of $\xi$. This is consistent with the special log case considered by Cole and Obstfeld (1991). 
Second, with recursive preferences, agents have a preference for the variance of their future utility, and hence their time-1 marginal utility depends on the time- 1 level of their utility. If agents prefer early resolution of uncertainty $\left(0>\theta^{i}>-\infty\right)$, a higher relative future utility implies a lower relative marginal utility, and hence a lower share of allocated resources $\left(u_{1}^{1}(\xi)>u_{1}^{2}(\xi) \rightarrow S_{1}(\xi)<S_{0}\right)$. Because of the dependence of $S_{1}$ on future utility levels, $u_{1}^{1}(\xi)-u_{1}^{2}(\xi)$, the shock $\xi$ prompts a reallocation at time 1 .

\section{Approximate solution}

Define $\frac{\lambda_{1}}{1-\lambda_{2}}=\kappa_{1}$ and $\frac{\lambda_{2}}{1-\lambda_{1}}=\kappa_{2}$. At time $1, \xi$ is known and the optimal allocations are

$$
X_{1}^{1}=\frac{S_{1} \kappa_{1}}{1+S_{1} \kappa_{1}} e^{\xi}, \quad X_{1}^{2}=\frac{1}{1+S_{1} \kappa_{1}} e^{\xi}
$$

and

$$
Y_{1}=\frac{\frac{S_{1}}{\kappa_{2}}}{1+\frac{S_{1}}{\kappa_{2}}} e^{-l \xi}, \quad Y_{1}^{2}=\frac{1}{1+\frac{S_{1}}{\kappa_{2}}} e^{-l \xi} .
$$

Since the allocations are a nonlinear function of $S_{1}(\xi)$, we log linearize them with respect to $s \equiv \log S_{1}$ around $\bar{s}=s_{0}$ and obtain

$$
\begin{aligned}
& \log \left(\frac{\kappa_{1} e^{s}}{1+\kappa_{1} e^{s}}\right)=\log \left(\frac{\kappa_{1} e^{\bar{s}}}{1+\kappa_{1} e^{\bar{s}}}\right)+\frac{1}{1+\kappa_{1} e^{\bar{s}}}(s-\bar{s}), \\
& \log \left(\frac{1}{1+\kappa_{1} e^{s}}\right)=\log \left(\frac{1}{1+\kappa_{1} e^{\bar{s}}}\right)-\frac{\kappa_{1} e^{\bar{s}}}{1+\kappa_{1} e^{\bar{s}}}(s-\bar{s}), \\
& \log \left(\frac{\frac{e^{s}}{\kappa_{2}}}{1+\frac{e^{s}}{\kappa_{2}}}\right)=\log \left(\frac{\frac{e^{\bar{s}}}{\kappa_{2}}}{1+\frac{e^{\bar{s}}}{\kappa_{2}}}\right)+\frac{1}{1+\frac{e^{\bar{s}}}{\kappa_{2}}}(s-\bar{s}), \\
& \log \left(\frac{1}{1+\frac{e^{s}}{\kappa_{2}}}\right)=\log \left(\frac{1}{1+\frac{e^{\bar{s}}}{\kappa_{2}}}\right)-\frac{\frac{e^{\bar{s}}}{\kappa_{2}}}{1+\frac{e^{\bar{s}}}{\kappa_{2}}}(s-\bar{s})
\end{aligned}
$$

and hence

$$
\begin{aligned}
\log C_{1}^{1}= & \lambda_{1}\left[\log \left(\frac{\kappa_{1} e^{\bar{s}}}{1+\kappa_{1} e^{\bar{s}}}\right)+\frac{1}{1+\kappa_{1} e^{\bar{s}}}(s-\bar{s})+\xi\right] \\
& +\left(1-\lambda_{1}\right)\left[\log \left(\frac{\frac{e^{\bar{s}}}{\kappa_{2}}}{1+\frac{e^{\bar{s}}}{\kappa_{2}}}\right)+\frac{1}{1+\frac{e^{\bar{s}}}{\kappa_{2}}}(s-\bar{s})-l \xi\right],
\end{aligned}
$$




$$
\begin{aligned}
\log C_{1}^{2}= & \left(1-\lambda_{2}\right)\left[\log \left(\frac{1}{1+\kappa_{1} e^{\bar{s}}}\right)-\frac{\kappa_{1} e^{\bar{s}}}{1+\kappa_{1} e^{\bar{s}}}(s-\bar{s})+\xi\right] \\
& +\lambda_{2}\left[\log \left(\frac{1}{1+\frac{e^{\bar{s}}}{\kappa_{2}}}\right)-\frac{\frac{e^{\bar{s}}}{\kappa_{2}}}{1+\frac{e^{\bar{s}}}{\kappa_{2}}}(s-\bar{s})-l \xi\right] .
\end{aligned}
$$

We can now write

$$
\begin{aligned}
& u_{1}^{1}=\log C_{1}^{1}=\bar{u}_{1}^{1}+\lambda_{u_{1}^{1}}^{\xi} \xi, \\
& u_{1}^{2}=\log C_{1}^{2}=\bar{u}_{1}^{2}+\lambda_{u_{1}^{2}}^{\xi} \xi,
\end{aligned}
$$

where

$$
\begin{aligned}
\lambda_{u_{1}^{1}}^{\xi} \equiv & {\left[\frac{\lambda_{1}}{1+\kappa_{1} e^{\bar{s}}}+\frac{1-\lambda_{1}}{1+\frac{e^{\bar{s}}}{\kappa_{2}}}\right] \lambda_{s}^{\xi}+\lambda_{1}-l+\lambda_{1} l, } \\
\lambda_{u_{1}^{2}}^{\xi} \equiv & {\left[-\left(1-\lambda_{2}\right) \frac{\kappa_{1} e^{\bar{s}}}{1+\kappa_{1} e^{\bar{s}}}-\lambda_{2} \frac{\frac{e^{\bar{s}}}{\kappa_{2}}}{1+\frac{e^{\bar{s}}}{\kappa_{2}}}\right] \lambda_{s}^{\xi}+\left(1-\lambda_{2}\right)-\lambda_{2} l, }
\end{aligned}
$$

and

$$
s-\bar{s}=\operatorname{const}(\bar{s})+\lambda_{s}^{\xi} \cdot \xi .
$$

The elasticity $\lambda_{s}^{\xi}$ can be found taking into account equation (A.5):

$$
\lambda_{s}^{\xi}=\frac{\lambda_{u_{1}^{1}}^{\xi}}{\theta_{1}}-\frac{\lambda_{u_{1}^{2}}^{\xi}}{\theta_{2}} .
$$

Specifically, by combining equations (A.8)-(A.9), we have

$$
\lambda_{s}^{\xi}=\frac{\frac{1}{\theta_{1}}\left[(1+l) \lambda_{1}-l\right]+\frac{1}{\theta_{2}}\left[(1+l) \lambda_{2}-1\right]}{1-\frac{1}{\theta_{1}}\left[\frac{\lambda_{1}}{1+\kappa_{1} e^{\bar{s}}}+\frac{1-\lambda_{1}}{1+\frac{e^{\bar{s}}}{\kappa_{2}}}\right]-\frac{1}{\theta_{2}}\left[\left(1-\lambda_{2}\right) \frac{\kappa_{1} e^{\bar{s}}}{1+\kappa_{1} e^{\bar{s}}}+\lambda_{2} \frac{\frac{e^{\bar{s}}}{\kappa_{2}}}{1+\frac{e^{\bar{s}}}{\kappa_{2}}}\right]} .
$$

The constant can be recovered again from equation (A.5) accounting for second-order terms:

$$
\operatorname{const}(\bar{s})=\frac{1}{2} \sigma^{2}\left[\left(\frac{\lambda_{u_{1}^{2}}^{\xi}}{\theta_{2}}\right)^{2}-\left(\frac{\lambda_{u_{1}^{1}}^{\xi}}{\theta_{1}}\right)^{2}\right]
$$




\section{Decentralization}

Let the budget constraints of agent 1 and agent 2 be

$$
\begin{array}{r}
X_{1}^{1}+p \cdot Y_{1}^{1}+\int_{\xi} Q(\xi) A_{2}^{1}(\xi)=e^{\xi}+A_{1}^{1}, \\
X_{1}^{2}+p \cdot Y_{1}^{2}+\int_{\xi} Q(\xi) A_{2}^{2}(\xi)=e^{-l \xi}+A_{1}^{2},
\end{array}
$$

where $p$ is the price of good $Y$ relative to good $X, Q(\xi)$ is the price of an Arrow-Debreu security that pays one unit of good $X$ (the numeraire) contingent on the realization of $\xi$ at date 2 , and $A_{i}^{j}$ are the holdings of such security of agent $j$ at date $i$. By market clearing, $A_{i}^{1}+A_{i}^{2}=0, \forall i \in\{1,2\}$.

Define $\bar{\xi}$ as the specific value of the state in which there is no reallocation effect, that is, $s=\bar{s}$. Under our approximation,

$$
\bar{\xi}=-\frac{\operatorname{const}(\bar{s})}{\lambda_{S}^{\xi}} .
$$

This value is an important threshold for the portfolio allocation of our two agents in the companion decentralized economy. In particular, at time 0 , agent 1 buys ArrowDebreu securities paying a positive payoff for $\xi<\bar{\xi}$, and sells Arrow-Debreu securities paying when $\xi>\bar{\xi}$. Since these assets are available in zero net supply, the exact opposite holds for agent 2 . The probability of agent 1 receiving a positive transfer is $F(\bar{\xi})$, where $F$ denotes the cumulative distribution function of $\xi$. When $\bar{\xi}>0$, agent 1 has a higher probability of being a net receiver of resources than agent 2 .

\section{Special cases}

In the special case in which both agents have the same risk-sensitivity parameter, $\theta_{1}=$ $\theta_{2}=\theta$, and the same exposure to the underlying shock, $l=1$, we obtain

$$
\lambda_{s}^{\xi}=\frac{\frac{2}{\theta}\left(\lambda_{1}+\lambda_{2}-1\right)}{1-\frac{1}{\theta}\left[\frac{\lambda_{1}}{1+\kappa_{1} e^{\bar{s}}}+\frac{1-\lambda_{1}}{1+\frac{e^{\bar{s}}}{\kappa_{2}}}+\left(1-\lambda_{2}\right) \frac{\kappa_{1} e^{\bar{s}}}{1+\kappa_{1} e^{\bar{s}}}+\lambda_{2} \frac{\frac{e^{\bar{s}}}{\kappa_{2}}}{1+\frac{e^{\bar{s}}}{\kappa_{2}}}\right]}
$$

and $\lambda_{S}^{\xi}<0$ if $\theta<-\infty$ and $\lambda_{1}+\lambda_{2}>1$, that is, the ratio of the pseudo-Pareto weights is "countercyclical," meaning that a smaller share of resources is assigned to agent 1 when there is relative abundance of good 1 . In this case,

$$
\operatorname{const}(\bar{s})=\frac{\sigma^{2}}{2 \theta^{2}}\left[\left(\lambda_{u_{1}^{2}}^{\xi}\right)^{2}-\left(\lambda_{u_{1}^{1}}^{\xi}\right)^{2}\right] .
$$




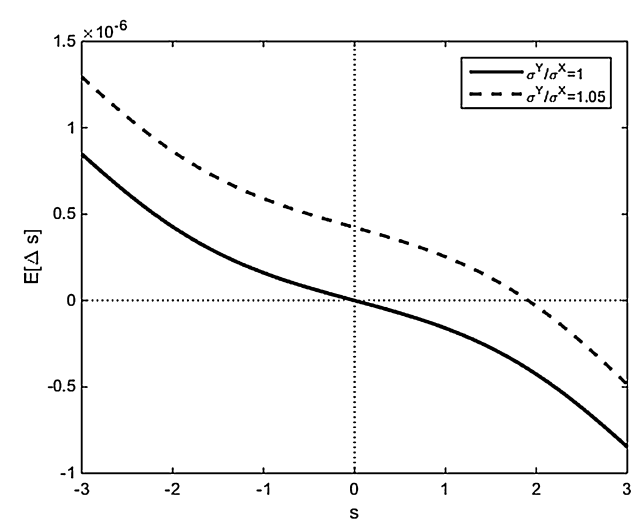

(a) Heterogenous volatility

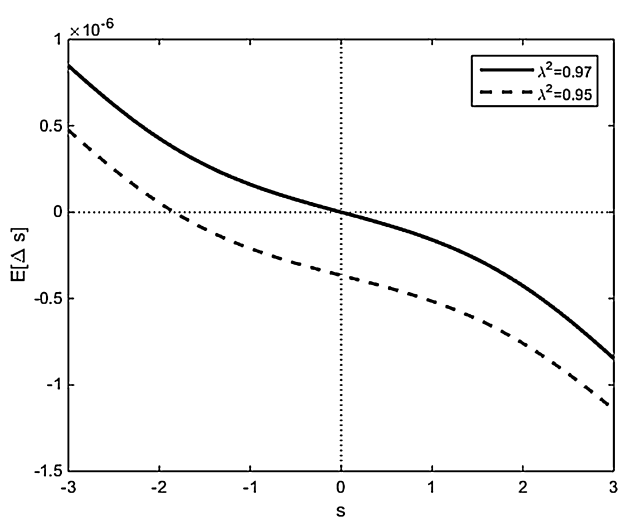

(b) Heterogenous degree of preference for the two goods

Figure AF-1. Asymmetric calibrations in the 2-period model. All results are based on the two-period model developed in the Appendix. All common parameters are set as in our reference calibration detailed in Section 5. We vary one parameter at a time, leaving the others unchanged.

Let us also assume that $\lambda_{1}=\lambda_{2}=\lambda>1 / 2$. In this case, we can derive the following results:

$$
\begin{aligned}
\lim _{s \rightarrow-\infty} \lambda_{s}^{\xi} & =4 \frac{(2 \lambda-1)}{\theta}<0, \\
\lim _{\bar{s} \rightarrow-\infty} \operatorname{const}(\bar{s}) & =\frac{\sigma^{2}}{2 \theta^{2}} \lambda_{s}^{\xi}\left[\lambda_{s}^{\xi}-2(2 \lambda-1)\right]>0, \\
\lim _{\bar{s} \rightarrow-\infty} \bar{\xi} & =(2 \lambda-1) \frac{\sigma^{2}}{\theta(\theta-1)}>0 .
\end{aligned}
$$

Let $F(\xi)$ be the cumulative distribution function of $\xi$. Conditional on $\bar{s} \rightarrow-\infty$, the probability of country 1 to receive a higher share of resources in period 1 is $F(\bar{\xi})$, and hence it increases with (i) a stronger degree of preference for one of the two goods $(\lambda)$, (ii) larger fundamental risk $\left(\sigma^{2}\right)$, and (iii) stronger risk sensitivity $(\theta)$.

Figure AF-1, depicts const $(\bar{s})$ (defined in equation (A.11)) as a function of $\bar{s}$ in two scenarios with asymmetric calibrations.

\section{Appendix B: Proofs}

\section{B. 1 Proof of Proposition 1}

Proof of Proposition 1. Define

$$
\begin{aligned}
& \varphi(z, S)=\frac{1}{\theta} \log c_{1}(z, S)-\frac{1}{\theta} \log c_{2}(z, S), \\
& \Phi(z, S)=\frac{1}{\theta} U_{1}(z, S)-\frac{1}{\theta} U_{2}(z, S), \\
& F(z, S)=\delta \log \sum_{z^{\prime}} \pi\left(z^{\prime}\right) \exp \left\{\frac{U_{1}\left(z^{\prime}, S^{\prime}\right)}{\theta}\right\}-\delta \log \sum_{z^{\prime}} \pi\left(z^{\prime}\right) \exp \left\{\frac{U_{2}\left(z^{\prime}, S^{\prime}\right)}{\theta}\right\} .
\end{aligned}
$$


Note that $\Phi(z, S)=\varphi(z, S)+F(z, S)$. Let $k=\lambda_{1} /\left(1-\lambda_{1}\right)$ and let $X(z)$ and $Y(z)$ be the endowments of the two goods in state $s$. Then, by the symmetry of the preferences, the optimal allocations imply

$$
\varphi(z, S)=\frac{1}{\theta}\left[\left(2 \lambda_{1}-1\right) \log \left(\frac{X(z)}{Y(z)}\right)+\log S+\left(1-2 \lambda_{1}\right) \log (1+k S)-\left(1-2 \lambda_{1}\right) \log (k+S)\right] .
$$

Since $\theta<0$, if $\lambda_{1}>1 / 2$ it follows that $\varphi(z, S)$ is always: 1) decreasing in $X(z) / Y(z)$ and 2) decreasing in $S$. Furthermore, $F(s, S)$ is decreasing in $S$, since $U_{1}(z, S)\left(U_{2}(z, S)\right)$ is increasing (decreasing) in $S$, by the optimality of the social planner problem. Therefore, it has to be the case that $\Phi(z, S)$ is decreasing in $S$.

Take two states, $a$ and $b$, such that $X^{\prime}(a) / Y^{\prime}(a)<X^{\prime}(b) / Y^{\prime}(b)$, and let $S_{a}^{\prime}=f_{S}(a, S)$ and $S_{b}^{\prime}=f_{S}(b, S)$ be their respective ratios of Pareto weights. It is possible to characterize the ratio:

$$
\frac{S_{b}^{\prime}}{S_{a}^{\prime}}=\exp \left\{\Phi\left(b, S_{b}^{\prime}\right)-\Phi\left(a, S_{a}^{\prime}\right)\right\}
$$

Since $\Phi(z, S)$ is decreasing in $z$ holding $S$ fixed, it follows that $\exp \left\{\Phi\left(b, S_{a}^{\prime}\right)-\Phi(a\right.$, $\left.\left.S_{a}^{\prime}\right)\right\}<1$. Hence, if $X^{\prime}(a) / Y^{\prime}(a)<X^{\prime}(b) / Y^{\prime}(b)$, it is never optimal to set $S_{a}^{\prime}=S_{b}^{\prime}$.

Additionally, the fact that $\Phi(s, S)$ is decreasing in $S$ implies that

$$
1>\exp \left\{\Phi\left(b, S_{a}^{\prime}\right)-\Phi\left(a, S_{a}^{\prime}\right)\right\}>\left\{\Phi\left(b, S_{b}^{\prime}\right)-\Phi\left(a, S_{a}^{\prime}\right)\right\} .
$$

It follows that $S_{b}^{\prime}<S_{a}^{\prime}$.

\section{B.2 Proof of Proposition 2}

We proceed in steps to provide a proof to Proposition 2. Specifically, we state and prove four propositions, which, combined, yield the proof of Proposition 2. Throughout our exposition of these propositions, we utilize the example proposed in main text, in order to better illustrate the content of each statement.

We start by stating the following decomposition of the Pareto weights, the proof for which follows directly from Anderson (2005). The rest of this section will consist of propositions aimed at signing the covariance that appears on the right-hand side of this decomposition. This will enable us to establish the relevant properties concerning the ergodicity and mean reversion of the distribution of Pareto weights.

Proposition A4. The ratio of Pareto weights can be decomposed as

$$
E\left[S^{\prime} \mid S\right]=S-\frac{\operatorname{cov}\left[\exp \left\{U_{2}^{\prime} / \theta\right\}, S^{\prime} \mid S\right]}{E\left[\exp \left\{U_{2}^{\prime} / \theta\right\} \mid S\right]},
$$

where $E\left[S^{\prime} \mid S\right]$ denotes the expectation of $S^{\prime}$ conditional on s being the current state, and

$$
\operatorname{cov}\left[\exp \left\{U_{2}^{\prime} / \theta\right\}, S^{\prime} \mid S\right]
$$

denotes the covariance between $\exp \left\{U_{2}^{\prime} / \theta\right\}$ and $S^{\prime}$ conditional on the current $S$. 
Proof. See Anderson (2005).

The next proposition draws a sharp contrast between a representative-agent economy and our setting with multiple agents and goods. In a representative-agent economy, the consumer enjoys a higher utility in the states of the world in which the supply of the most-preferred good is more abundant. In the two agent economy that we consider in this paper, for any two symmetric states there exists a finite ratio of Pareto weights below which the ranking of the future utility functions across states is reversed.

Proposition A5. For any two symmetric states $z_{i}$ and $z_{-i}$, such that $X\left(z_{i}\right)>Y\left(z_{i}\right)$, there exists a finite $\widetilde{S}_{1}^{i}<1$ such that $U_{1}\left(z_{i}, f_{S}\left(z_{i}, \widetilde{S}_{1}^{i}\right)\right)=U_{1}\left(z_{-i}, f_{S}\left(z_{-i}, \widetilde{S}_{1}^{i}\right)\right)$, where $f_{S}(\cdot, \cdot)$ is defined in (10), and $U_{1}\left(z_{i}, f_{S}\left(z_{i}, S_{1}^{i}\right)\right)<U_{1}\left(z_{-i}, f_{S}\left(z_{-i}, S_{1}^{i}\right)\right), \forall S_{1}^{i}<\widetilde{S}_{1}^{i}$.

Proof. Let $S_{i}^{\prime}=f_{S}\left(z_{i}, S\right)$ and $S_{-i}^{\prime}=f_{S}\left(z_{-i}, S\right)$ be the ratios of Pareto weights when tomorrow's symmetric states are $z_{i}$ and $z_{-i}$, respectively. Using equation (9), the ratio between $S_{i}^{\prime}$ and $S_{-i}^{\prime}$ is

$$
\frac{S_{i}^{\prime}}{S_{-i}^{\prime}}=\exp \left\{\frac{U_{1}\left(z_{i}^{\prime}, S_{i}^{\prime}\right)-U_{1}\left(z_{-i}^{\prime}, S_{-i}^{\prime}\right)}{\theta}\right\} / \exp \left\{\frac{U_{2}\left(z_{i}^{\prime}, S_{i}^{\prime}\right)-U_{2}\left(z_{-i}^{\prime}, S_{-i}^{\prime}\right)}{\theta}\right\} .
$$

Rearranging, we have

$$
U_{1}\left(z_{i}^{\prime}, S_{i}^{\prime}\right)-U_{1}\left(z_{-i}^{\prime}, S_{-i}^{\prime}\right)=U_{2}\left(s_{i}^{\prime}, S_{i}^{\prime}\right)-U_{2}\left(z_{-i}^{\prime}, S_{-i}^{\prime}\right)+\theta\left[\log S_{i}^{\prime}-\log S_{-i}^{\prime}\right] .
$$

We shall characterize the limit of the left-hand side of equation (B.18) for $\mu_{1}$ that tends to zero. First, notice that consumption bias $\left(\lambda_{1}>1 / 2\right)$ implies that

$$
\lim _{\mu_{1} \rightarrow 0} U_{2}\left(z_{i}^{\prime}, S_{i}^{\prime}\right)-U_{2}\left(z_{-i}^{\prime}, S_{-i}^{\prime}\right)<0 .
$$

Then, notice that equation (C.35) and the fact that the planner's problem is twice continuously differentiable imply the continuity of the $f_{S}$ function with respect to $S$. Also, at $\mu_{1}=0, S_{i}^{\prime}=S_{-i}^{\prime}=0$. Hence

$$
\lim _{\mu_{1} \rightarrow 0} S_{i}^{\prime}=\left.S_{i}^{\prime}\right|_{\mu_{1}=0}=\left.S_{-i}^{\prime}\right|_{\mu_{1}=0}=\lim _{\mu_{1} \rightarrow 0} S_{-i}^{\prime},
$$

which implies that

$$
\lim _{\mu_{1} \rightarrow 0} \log \left(S_{i}^{\prime}-\log S_{-i}^{\prime}\right)=0 .
$$

Combining (B.19) and (B.20) into (B.18), we obtain that

$$
\lim _{\mu_{1} \rightarrow 0} U_{1}\left(z_{i}^{\prime}, S_{i}^{\prime}\right)-U_{1}\left(z_{-i}^{\prime}, S_{-i}^{\prime}\right)<0 .
$$

Since $\lambda_{1}>1 / 2$, it follows that

$$
\lim _{\mu_{1} \rightarrow 1} U_{1}\left(z_{i}, S^{\prime}\right)-U_{1}\left(z_{-i}, S^{\prime}\right)>0 .
$$

Combining (B.21) and (B.22) concludes the proof. 


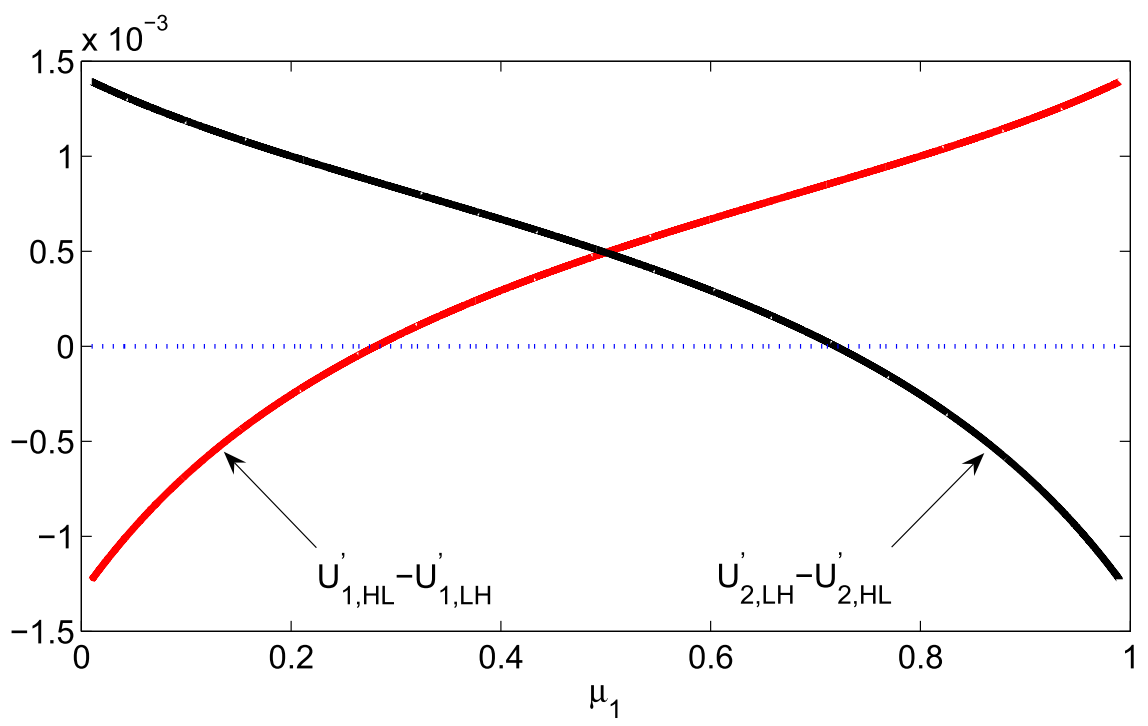

Figure AF-2. Difference of continuation utilities in symmetric states. This figure depicts the difference of the continuation utilities in the states of high supply of good $X$-low supply of $\operatorname{good} Y, U_{i, H L}^{\prime}$, and low supply of good $X$-high supply of good $Y, U_{i, L H}^{\prime}$, for $i=1,2$. The red line refers to agent 1 , the black line to agent 2 .

Corollary 1. For any two symmetric states $z_{i}$ and $z_{-i}$, such that $X\left(z_{i}\right)>Y\left(z_{i}\right)$, there exists a finite $\tilde{S}_{2}^{i}>1$ such that $U_{2}\left(z_{i}, f_{S}\left(z_{i}, \widetilde{S}_{2}^{i}\right)\right)=U_{2}\left(z_{-i}, f_{S}\left(z_{-i}, \widetilde{S}_{2}^{i}\right)\right)$, where $f_{S}(\cdot, \cdot)$ is defined in (10), and $U_{2}\left(z_{i}, f_{S}\left(z_{i}, S_{2}^{i}\right)\right)>U_{2}\left(z_{-i}, f_{S}\left(z_{-i}, S_{2}^{i}\right)\right), \forall S_{2}^{i}>\widetilde{S}_{2}^{i}$.

We illustrate the content of the preceding proposition and corollary in Figure AF-2, which depicts the differences of the continuation utilities in the two states of unequal supply of the two goods for the example discussed in Section 3.2 of the main text.

When the Pareto weight attached to agent 1 (agent 2) is approaching 1, the continuation utility for the state of abundant supply of good $X(\operatorname{good} Y)$ is higher than the continuation utility for the state of scarce supply of good $X$. However, as suggested by Proposition A5, there exists a $\tilde{\mu}_{1}<1 / 2\left(1-\tilde{\mu}_{1}<1 / 2\right)$ past which the ranking of the continuation utilities is reversed.

We are now ready to characterize the sign of the covariance term in (B.17). The following definition of conditional covariance in symmetric states is useful in establishing an upper bound on the last term of equation (B.17).

Definition 2 (Covariance of Symmetric States). Let $z_{i}, z_{-i} \in \mathcal{N}$ be symmetric states. The conditional covariance between two random variables $h$ and $g$ valued on $\left\{z_{i}, z_{-i}\right\}$ is

$$
\operatorname{cov}_{i,-i}[h, g \mid S]=\sum_{l=\{i,-i\}} p\left(z_{l}\right) h\left(z_{l}\right) g\left(z_{l}\right)-\left(\sum_{l=\{i,-i\}} p\left(z_{l}\right) h\left(z_{l}\right)\right)\left(\sum_{l=\{i,-i\}} p\left(z_{l}\right) g(l)\right),
$$

where $p\left(z_{l}\right) \equiv \pi\left(z_{l}\right) /\left(\pi\left(z_{i}\right)+\pi\left(z_{-i}\right)\right)$. 
Proposition A6. Let $\overline{\operatorname{cov}}$ be the sum of the conditional covariances between $\exp \left\{U_{2}^{\prime} / \theta\right\}$ and $S^{\prime}$ across all symmetric states:

$$
\overline{\operatorname{cov}}=\sum_{i} \operatorname{cov}_{i,-i}\left[\exp \left\{U_{2}^{\prime} / \theta\right\}, S^{\prime} \mid S\right] .
$$

If $\overline{\operatorname{cov}}<0$, then

$$
\operatorname{cov}\left[\exp \left\{U_{2}^{\prime} / \theta\right\}, S^{\prime} \mid S\right]<0 .
$$

Proof. By the definition of conditional covariance,

$$
\begin{aligned}
\operatorname{cov}\left[\exp \left\{U_{2}^{\prime} / \theta\right\}, S^{\prime} \mid S\right]= & \sum_{j=1}^{n} \pi(j) \exp \left\{U_{2}^{\prime}(j) / \theta\right\} S^{\prime}(j) \\
& -\left[\sum_{j=1}^{n} \pi(j) \exp \left\{U_{2}^{\prime}(j) / \theta\right\}\right]\left[\sum_{j=1}^{n} \pi(j) S^{\prime}(j)\right] \\
= & \bar{\pi} \sum_{i} \operatorname{cov}_{i,-i}\left[\exp \left\{U_{2}^{\prime} / \theta\right\}, S^{\prime} \mid S\right]+\operatorname{cov}_{\mathrm{eq}}\left[\exp \left\{U_{2}^{\prime} / \theta\right\}, S^{\prime} \mid S\right] \\
& -\left(\sum_{i} \widetilde{\pi}(i) \exp \left\{U_{2}^{\prime} / \theta\right\}\right)\left(\sum_{i} \widetilde{\pi}(i) S^{\prime}\right),
\end{aligned}
$$

where $\bar{\pi}$ is a nonnegative scalar smaller than one; $\sum_{i} \operatorname{cov}_{i,-i}\left[\exp \left\{U_{2}^{\prime} / \theta\right\}, S^{\prime} \mid S\right]$ is the conditional covariance across all symmetric states; $\operatorname{cov}_{\mathrm{eq}}\left[\exp \left\{U_{2}^{\prime} / \theta\right\}, S^{\prime} \mid S\right]$ is the conditional covariance across all states of equal supply; and $\{\widetilde{\pi}(i)\}_{i=1}^{n}$ and $\{\widetilde{\pi}(i)\}_{i=1}^{n}$ are nonnegative sequences of scalars.

We know from Proposition 1 that the optimal choice of Pareto weights is identical across all states in which the supply of the two goods is the same. This implies that

$$
\operatorname{cov}_{\mathrm{eq}}\left[\exp \left\{U_{2}^{\prime} / \theta\right\}, S^{\prime} \mid S\right]=0 .
$$

We can also conclude that the last term in equation (B.24) is nonnegative, being the product of the sums of nonnegative terms.

Therefore, we can state that

$$
\operatorname{cov}\left[\exp \left\{U_{2}^{\prime} / \theta\right\}, S^{\prime} \mid S\right] \leq \bar{\pi} \sum_{i} \operatorname{cov}_{i,-i}\left[\exp \left\{U_{2}^{\prime} / \theta\right\}, S^{\prime} \mid S\right],
$$

which concludes the proof.

The sign of $\overline{c o v}$ is key. Suppose that the current ratio of Pareto weights is in the region between zero and $\min _{i}\left\{\widetilde{S}_{2}^{i}\right\}$ as defined in Corollary 1 . For any two symmetric states, the utility of agent 2 is going to be larger when the supply of good $Y$ is relatively more abundant. Since the parameter $\theta$ is negative, the ranking of $U_{2} / \theta$ is reversed. Proposition 1 suggests that the ratio of Pareto weights is larger when the supply of good $Y$ is larger. 


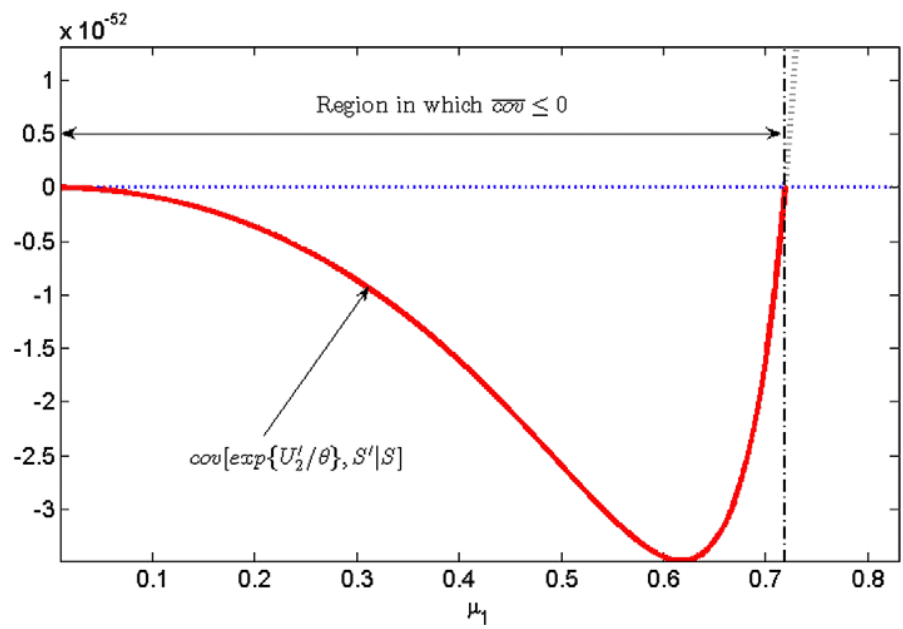

Figure AF-3. Upper bound on the conditional covariance between $\exp \left\{U_{2}^{\prime} / \theta\right\}$ and $S^{\prime}$ as a function of the current Pareto weight.

Therefore, we can conclude that, in this region, $\overline{\operatorname{cov}}$, and hence the covariance term in (B.17), is negative. This argument is summarized in Figure AF-3.

The next proposition plays a crucial role in our proof for the existence of a welldefined ergodic distribution of the Pareto weights. We focus on the properties of the left tail of the stochastic process $S^{\prime}=\frac{\mu_{1}^{\prime}}{1-\mu_{1}^{\prime}}=f_{S}\left(z^{\prime}, S\right)$ and base our proof on all the other propositions developed so far.

Proposition A7. The stochastic process $\widehat{S}(S):=\min \left(S, \min _{i}\left\{\widetilde{S}_{2}^{i}\right\}\right)$, where $\widetilde{S}_{2}^{i}$ is defined as in Corollary 1 is a submartingale.

Proof. Follows directly from all the propositions in this Appendix.

We conclude this section with the proof of Proposition 2.

Proof of Proposition 2. We know from Proposition A7 that the stochastic process

$$
\widehat{S}(S)=\min \left(S, \min _{i}\left\{\widetilde{S}_{2}^{i}\right\}\right)
$$

is a submartingale. Since a bounded submartingale cannot converge almost surely to its lower bound (see Sciubba (2005)), then $\widehat{S}(S)$ cannot converge almost surely to 0 .

We prove the rest of the proposition by contradiction. Denote by $\Omega=\mathrm{X}_{t=1}^{\infty} z_{t}$ the set of sample paths of endowments, with representative element $\omega=\left(z_{1}, z_{2}, \ldots, z_{t}, \ldots\right)$. Given the initial Pareto weight ratio $S_{0}=1$ and the transition dynamics $S^{\prime}=f_{S}\left(z^{\prime}, S\right)$, the ratio of Pareto weights at time $t$ can be written as a function of the sample path: $S_{t}=S_{t}(\omega)$.

Suppose Proposition 2 does not hold. Then there must exist a set of $\omega$ such that

$$
\operatorname{Pr}\left\{\omega \mid \lim _{t \rightarrow \infty} S_{t}(\omega)=0\right\}>0 .
$$


Denote by $Q=\left\{\omega \mid \lim _{t \rightarrow \infty} S_{t}(\omega)=0\right\}$ and consider the natural filtration on $Q: Q_{1} \subseteq Q_{2} \subseteq$ $\cdots \subseteq Q_{t} \subseteq \cdots \subseteq Q$. Recall that $\left\{z_{t}\right\}_{t=1}^{\infty}$ is i.i.d. As in Sciubba (2005), $Q_{t-1}$ does not restrict the original probability distribution of $z_{t}$, and hence we have that $\operatorname{Pr}\left\{z_{t}=z(i) \mid Q_{t-1}\right\}=$ $\pi(z(i))$. As a consequence, all the results that we have proved so far in this section apply with respect to the conditioning set $Q_{t-1}$. This means that $S_{t}$ is a bounded submartingale with respect to the conditioning information set $Q_{t-1}$. Since a bounded submartingale cannot converge almost surely to its lower bound, then $S_{t}$ cannot converge almost surely to 0 on $Q$. This contradicts the definition of $Q$. Hence, $S_{t}$ cannot converge to 0 with probability 1 . Equivalently, the probability that $S_{t}$ converges to 0 is null.

Note that $\mu_{1}(\widehat{S})=\frac{\widehat{S}}{1+\widehat{S}}$, that is, $\mu_{1}(\widehat{S})$ is a nonnegative, monotonically increasing function of $\widehat{S}$. It follows that $\mu_{1}$ cannot also converge to 0 almost surely. By repeating all the proofs in the paper for $\mu_{2}=1-\mu_{1}$, it follows by symmetry that $\mu_{2}$ cannot converge to 0 almost surely. Equivalently, $\mu_{1}$ cannot converge to 1 almost surely either.

\section{B.3 Proof of Proposition 3}

This section reports the proof of the proposition on the mean-reversion property of the Pareto weight.

Proof of Proposition 3. We prove the three statements in Proposition 3 in the same order in which they appear in the proposition's claim.

1. We show that only $\mu_{1}=1 / 2$ is such that $E\left[\mu_{1}^{\prime} \mid S\right]=\mu_{1}$.

After imposing a symmetrical calibration, the recursive definition of the ratio of Pareto weights derived in the paper becomes

$$
\frac{\mu_{1}^{\prime}}{1-\mu_{1}^{\prime}}=\frac{\mu_{1}}{1-\mu_{1}} \cdot \frac{\exp \left\{U_{1}\left(z^{\prime}, S^{\prime}\right) / \theta\right\}}{\sum_{z^{\prime}} \pi\left(z^{\prime}\right) \exp \left\{U_{1}\left(z^{\prime}, S^{\prime}\right) / \theta\right\}} \frac{\sum_{z^{\prime}} \pi\left(z^{\prime}\right) \exp \left\{U_{2}\left(z^{\prime}, S^{\prime}\right) / \theta\right\}}{\exp \left\{U_{2}\left(z^{\prime}, S^{\prime}\right) / \theta\right\}} .
$$

By rearranging and taking the conditional expectation operator, we obtain

$$
\begin{aligned}
E\left[\mu_{1}^{\prime} \mid S\right]= & \mu_{1}-\mu_{1} \cdot \frac{\operatorname{cov}\left[\mu_{1}^{\prime}, \exp \left\{U_{1}^{\prime} / \theta\right\} \mid S\right]}{E\left[\exp \left\{U_{1}^{\prime} / \theta\right\} \mid S\right]} \\
& -\left(1-\mu_{1}\right) \cdot \frac{\operatorname{cov}\left[\mu_{1}^{\prime}, \exp \left\{U_{2}^{\prime} / \theta\right\} \mid S\right]}{E\left[\exp \left\{U_{2}^{\prime} / \theta\right\} \mid S\right]} .
\end{aligned}
$$

For each $\mu_{1}=\mu_{1}^{*}$ such that $E\left[\mu_{1}^{\prime} \mid S\right]=\mu_{1}^{*}$, equation (B.26) implies that

$$
\begin{aligned}
\mu_{1}^{*}= & \frac{\operatorname{cov}\left[\mu_{1}^{\prime}, \exp \left\{U_{2}^{\prime} / \theta\right\} \mid S\right]}{E\left[\exp \left\{U_{2}^{\prime} / \theta\right\} \mid S\right]} \\
& /\left(\frac{\operatorname{cov}\left[\mu_{1}^{\prime}, \exp \left\{U_{2}^{\prime} / \theta\right\} \mid S\right]}{E\left[\exp \left\{U_{2}^{\prime} / \theta\right\} \mid S\right]}-\frac{\operatorname{cov}\left[\mu_{1}^{\prime}, \exp \left\{U_{1}^{\prime} / \theta\right\} \mid S\right]}{E\left[\exp \left\{U_{1}^{\prime} / \theta\right\} \mid S\right]}\right) .
\end{aligned}
$$


By symmetry, for each $\mu_{1}^{*}<1 / 2$, there must exist a $\mu_{1}^{* *}=1-\mu_{1}^{*}$ such that $E\left[\mu_{1}^{\prime} \mid S\right]=\mu_{1}^{* *}$. At $\mu_{1}^{* *}=1-\mu_{1}^{*}$, equation (B.26) implies that

$$
\begin{aligned}
\mu_{1}^{*}= & \frac{\operatorname{cov}\left[\mu_{1}^{\prime}, \exp \left\{U_{1}^{\prime} / \theta\right\} \mid S\right]}{E\left[\exp \left\{U_{1}^{\prime} / \theta\right\} \mid S\right]} \\
& /\left(\frac{\operatorname{cov}\left[\mu_{1}^{\prime}, \exp \left\{U_{1}^{\prime} / \theta\right\} \mid S\right]}{E\left[\exp \left\{U_{1}^{\prime} / \theta\right\} \mid S\right]}-\frac{\operatorname{cov}\left[\mu_{1}^{\prime}, \exp \left\{U_{2}^{\prime} / \theta\right\} \mid S\right]}{E\left[\exp \left\{U_{2}^{\prime} / \theta\right\} \mid S\right]}\right) .
\end{aligned}
$$

Combining equations (B.27) and (B.28), we obtain that at $\mu_{1}^{*}$ the following condition must hold:

$$
\frac{\operatorname{cov}\left[\mu_{1}^{\prime}, \exp \left\{U_{1}^{\prime} / \theta\right\} \mid S\right]}{E\left[\exp \left\{U_{1}^{\prime} / \theta\right\} \mid S\right]}=-\frac{\operatorname{cov}\left[\mu_{1}^{\prime}, \exp \left\{U_{2}^{\prime} / \theta\right\} \mid S\right]}{E\left[\exp \left\{U_{2}^{\prime} / \theta\right\} \mid S\right]} .
$$

By plugging condition (B.29) into equation (B.27) or (B.28), we conclude that the only $\mu_{1}^{*} \in(0,1)$ such that $E\left[\mu_{1}^{\prime} \mid S\right]=\mu_{1}^{*}$ is $\mu_{1}^{*}=1 / 2$.

2. We show that if $\mu_{1}>1 / 2$, then $E\left[\mu_{1}^{\prime} \mid S\right]<\mu_{1}$.

We know from Proposition A7 that the stochastic process $\widehat{S}(S)=\min \left(S, \min _{i}\left\{\tilde{S}_{2}^{i}\right\}\right)$, where $\min _{i}\left\{\widetilde{S}_{2}\right\}>1$ is a submartingale. Since, by definition, $\widehat{S}(S)=\frac{\mu_{1}(\widehat{S})}{1-\mu_{1}(\widehat{S})}$, and the stochastic process $\mu_{1}$ cannot converge to 1 (see Proposition 2), there must exist a $\mu_{1}^{+}>\frac{\min _{i} \widetilde{S}_{2}^{i}}{1+\min _{i} \widetilde{S}_{2}^{i}}>1 / 2$ such that $E\left[S^{\prime} \mid S^{+}\right]=S^{+}$, where $S^{+}=\frac{\mu_{1}^{+}}{1-\mu_{1}^{+}}$. Since $S=\frac{\mu_{1}}{1-\mu_{1}}$ is a convex function in $\mu_{1}$,

$$
\begin{aligned}
\frac{\mu_{1}^{+}}{1-\mu_{1}^{+}} & =E\left[S^{\prime} \mid S^{+}\right]=E\left[\frac{\mu_{1}^{\prime}}{1-\mu_{1}^{\prime}} \mid S^{+}\right] \\
& =\operatorname{cov}\left[\mu_{1}^{\prime}, \frac{1}{1-\mu_{1}^{\prime}} \mid S^{+}\right]+E\left[\mu_{1}^{\prime} \mid S^{+}\right] E\left[\frac{1}{1-\mu_{1}^{\prime}} \mid S^{+}\right] \\
& \geq \operatorname{cov}\left[\mu_{1}^{\prime}, \frac{1}{1-\mu_{1}^{\prime}} \mid S^{+}\right]+E\left[\mu_{1}^{\prime} \mid S^{+}\right] \frac{1}{1-E\left[\mu_{1}^{\prime} \mid S^{+}\right]} .
\end{aligned}
$$

Since $\mu_{1}^{\prime}$ and $\frac{1}{1-\mu_{1}^{\prime}}$ are both monotonically increasing functions of $\mu_{1}^{\prime}, \operatorname{cov}\left[\mu_{1}^{\prime}, \frac{1}{1-\mu_{1}^{\prime}} \mid S^{+}\right]>$ 0 . This implies that

$$
\begin{aligned}
\frac{\mu_{1}^{+}}{1-\mu_{1}^{+}} & \geq \operatorname{cov}\left[\mu_{1}^{\prime}, \frac{1}{1-\mu_{1}^{\prime}} \mid S^{+}\right]+E\left[\mu_{1}^{\prime} \mid S^{+}\right] \frac{1}{1-E\left[\mu_{1}^{\prime} \mid S^{+}\right]} \\
& >E\left[\mu_{1}^{\prime} \mid S^{+}\right] \frac{1}{1-E\left[\mu_{1}^{\prime} \mid S^{+}\right]}
\end{aligned}
$$

from which we obtain

$$
E\left[\mu_{1}^{\prime} \mid S^{+}\right]<\mu_{1}^{+} .
$$

Since we know that there exists only one $\mu_{1}^{*}=1 / 2$ such that $E\left[\mu_{1}^{\prime} \mid \frac{\mu_{1}^{*}}{1-\mu_{1}^{*}}\right]=\mu_{1}^{*}$, by the continuity of $f_{S}$ with respect to $S$, it must be the case that $E\left[\mu_{1}^{\prime} \mid S\right]<\mu_{1}, \forall \mu_{1} \in(1 / 2,1)$. 
3. We show that if $\mu_{1}<1 / 2$, then $E\left[\mu_{1}^{\prime} \mid S\right]>\mu_{1}$.

This proof mirrors the one that we just provided for $\mu_{1}>1 / 2$, and thus for parsimony we omit it here.

\section{Appendix C: Recursive Method And global SOlution}

Given the conditions in Ma (1993) and Ma (1996), the social planner's value function, denoted as $Q_{p}\left(z, \mu_{1}\right): \mathcal{N} \times[0,1] \rightarrow R$, satisfies the following functional equation proposed by Lucas and Stokey (1984), and Kan (1995):

$$
Q_{p}\left(z, \mu_{1}\right)=\max _{\left\{x_{i}, y_{i}, q_{i, z^{\prime}}\right\}_{i \in\{1,2\}, z^{\prime} \in \mathcal{N}}} \sum_{i=1}^{2} \mu_{i} \log W_{i}^{*}\left(z, c_{i},\left\{q_{i, z^{\prime}}\right\}_{z^{\prime}}\right)
$$

subject to

$$
\begin{aligned}
\mu_{2} & =1-\mu_{1}, \\
0 & \leq x_{1} \leq X(z), \quad 0 \leq x_{2} \leq X(z)-x_{1}, \\
0 & \leq y_{1} \leq Y(z), \quad 0 \leq y_{2} \leq Y(z)-y_{1}, \\
c_{i} & =\left(x_{i}\right)^{\lambda_{i}}\left(y_{i}\right)^{1-\lambda_{i}}, \quad \forall i=\{1,2\}, \\
0 & \leq \min _{\mu_{1}\left(z^{\prime}\right) \in[0,1]} Q_{p}\left(z^{\prime}, \mu_{1}\left(z^{\prime}\right)\right)-\mu_{1}\left(z^{\prime}\right) q_{1, z^{\prime}}-\left(1-\mu_{1}\left(z^{\prime}\right)\right) q_{2, z^{\prime}}, \quad \forall z^{\prime} \in \mathcal{N} .
\end{aligned}
$$

Remark Appendix C.1. Let Assumptions 1-4 hold. When $\mu_{i}=0$, interpret $\mu_{i} \log W_{i}^{*}(z$, $\left.c_{i},\left\{q_{i, z^{\prime}}\right\}_{z^{\prime}}\right)=0$. Since $0<X(z)<\infty$ and $0<Y(z)<\infty, \forall z \in \mathcal{N}$, it can be proved that there exists a unique bounded and continuous solution to (C.30)-(C.31). A feasible allocation is Pareto optimal if and only if it is generated recursively from the solution to (C.30)-(C.31).

Assumptions 1-4 are sufficient to apply the contraction mapping theorem to the planner's problem described by the system of equations (4)-(5). This result is parallel to those proposed by Lucas and Stokey (1984), Kan (1995), and Anderson (2005).

\section{IES $=1:$ A two-step approach}

It is convenient to use the definition of risk sensitive preferences in equation (1) to notice that the recursive planner's problem can be decomposed in two parts:

$$
Q_{p}\left(s, \mu_{1}\right)=Q_{p}^{A}\left(s, \mu_{1}\right)+Q_{p}^{B}\left(s, \mu_{1}\right)
$$

where $Q_{p}^{A}\left(s, \mu_{1}\right)$ is the static social planning problem

$$
Q_{p}^{A}\left(s, \mu_{1}\right) \equiv \max _{\left\{x_{i} \geq 0, y_{i} \geq 0\right\}_{i \in\{1,2\}}} \mu_{1}\left(1-\delta_{1}\right) \log c_{1}+\left(1-\mu_{1}\right)\left(1-\delta_{2}\right) \log c_{2}
$$


subject to:

$$
\begin{aligned}
X(z) & \geq x_{1}+x_{2}, \quad Y(z) \geq y_{1}+y_{2}, \\
c_{i} & =\left(x_{i}\right)^{\lambda_{i}}\left(y_{i}\right)^{1-\lambda_{i}}, \quad i=1,2 ;
\end{aligned}
$$

and $Q_{p}^{B}\left(s, \mu_{1}\right)$ is related to the allocation of future utilities

$$
\begin{aligned}
Q_{p}^{B}\left(z, \mu_{1}\right) \equiv & \max _{D_{z^{\prime}}} \mu_{1} \delta_{1} \log \left[\sum_{z^{\prime}} \exp \left\{\left(1-\gamma_{1}\right)\left(q_{2, z^{\prime}}+D_{z^{\prime}}\right)\right\} \pi_{s}\right] \\
& +\left(1-\mu_{1}\right) \delta_{2} \log \left[\sum_{z^{\prime}} \exp \left\{\left(1-\gamma_{2}\right) q_{2, z^{\prime}}\right\} \pi_{s}\right]
\end{aligned}
$$

subject to

$$
q_{2, z^{\prime}}=Q_{p}\left(z^{\prime},{\frac{d Q_{p}}{d \mu_{1}}}^{-1}\left(z^{\prime}, D_{z^{\prime}}\right)\right)-D_{z^{\prime}} \frac{d Q_{p}}{d \mu_{1}} \quad\left(z^{\prime}, D_{z^{\prime}}\right), \quad \forall z^{\prime} \in \mathcal{N}
$$

where $D_{z^{\prime}}$ is defined as the difference of the future utilities in state $z^{\prime}: D_{z^{\prime}} \equiv q_{1, z^{\prime}}-q_{2, z^{\prime}}$. Equation (C.34) defines the Pareto frontier and is obtained using equations (5)-(7). By equation (7), the first derivative of $Q_{p}$ is strictly increasing, hence, invertible. Equation (6) implies that:

$$
\mu_{1}(z)={\frac{d Q_{p}}{d \mu_{1}}}^{-1}\left(z, q_{1, z}-q_{2, z}\right)
$$

\section{Appendix D: Allocation as a function of Pareto weights}

Let $W_{t}^{i}=W\left(C_{t}^{i}, U_{t+1}^{i}\right)$ be the right-hand side of equation (14). If we denote the partial derivatives of the aggregator $W^{i}$ as

$$
W_{1, t}^{i}:=\frac{\partial W_{t}^{i}}{\partial C_{t}^{i}}, \quad W_{2, t}^{i}:=\frac{\partial W_{t}^{i}}{\partial U_{t+1}^{i}}
$$

then the stochastic discount factor is equal to

$$
M_{t+1}^{i}=\frac{W_{2, t}^{i} W_{1, t+1}^{i}}{W_{1, t}^{i}}, \quad \forall i=\{1,2\}
$$

The optimality condition for the allocation of good $X_{t}$ for $t=1,2, \ldots$ in each possible state is

$$
\mu_{0}^{h} \cdot\left(\prod_{j=0}^{t-1} W_{2, j}^{1}\right) \cdot W_{1, t}^{1} C_{t}^{1} \frac{\lambda_{1}}{x_{t}^{1}}=\frac{\left(1-\lambda_{2}\right)}{x_{t}^{2}} C_{t}^{2} W_{1, t}^{2} \cdot\left(\prod_{j=0}^{t-1} W_{2, j}^{2}\right) \cdot \mu_{0}^{2}
$$


Define the date $t$ Pareto weights as

$$
\begin{aligned}
\mu_{t}^{i} & =\mu_{0}^{i} \cdot\left(\prod_{j=0}^{t-1} W_{2, j}^{i}\right) \cdot W_{1, t}^{i} C_{t}^{i} \\
& =\mu_{t-1}^{i} \cdot W_{2, t-1}^{i} \cdot \frac{W_{1, t}^{i}}{W_{1, t-1}^{i}} \cdot \frac{C_{t}^{i}}{C_{t-1}^{i}}=\mu_{t-1}^{i} \cdot M_{t}^{i} \cdot \exp \left\{\Delta c_{t}^{i}\right\}, \quad \forall i \in\{h, f\} .
\end{aligned}
$$

It follows that equation (D.37) can be rewritten as

$$
\mu_{t}^{1} \cdot \frac{\lambda_{1}}{x_{t}^{1}}=\frac{\left(1-\lambda_{2}\right)}{x_{t}^{2}} \cdot \mu_{t}^{2} .
$$

Let $S_{t}:=\mu_{t}^{1} / \mu_{t}^{2}$. Then the optimality condition in equation (D.38) can be represented by the following system of recursive equations:

$$
\begin{aligned}
S_{t} \frac{\lambda_{1}}{x_{t}^{1}} & =\frac{\left(1-\lambda_{2}\right)}{x_{t}^{2}}, \\
S_{t} & =S_{t-1} \frac{M_{t}^{1} e^{\Delta c_{t}^{1}}}{M_{t}^{2} e^{\Delta c_{t}^{2}}},
\end{aligned}
$$

where

$$
M_{i, t+1}=\delta\left(\frac{C_{i, t+1}}{C_{i, t}}\right)^{-\frac{1}{\psi}}\left(\frac{U_{i, t+1}^{1-\gamma}}{E_{t}\left[U_{i, t+1}^{1-\gamma}\right]}\right)^{\frac{1 / \psi-\gamma}{1-\gamma}} .
$$

A similar first-order condition applies with respect to good $Y$.

\section{Approximation methods and errors}

We use perturbation methods to solve our system of equations and compute our policy functions using the dynare ++4.2 .1 package. All variables are expressed in log-units.

In Tables 1 and 2, we report statistics regarding the maximum cumulative approximation error (Cumul. ERR) for our relative pseudo-Pareto weights, that is, the key drivers of both consumption shares. The maximum is taken across agents, and we account for the fact that the relative Pareto weights are persistent and errors can accumulate over longer simulations.

More specifically, we construct recursively the following processes:

$$
\tilde{S}_{j, t}=\tilde{S}_{j, t-1} \cdot \frac{\hat{M}_{j, t}}{\hat{M}_{1, t}} \cdot\left(\frac{\hat{C}_{j, t} / \hat{C}_{j, t-1}}{\hat{C}_{1, t} / \hat{C}_{1, t-1}}\right), \quad \forall t \geq 1,
$$

where we adopt the "^" notation to indicate approximated variables. We initialize the recursion at different starting points, $\tilde{S}_{j, 0}$ and we then define the error for agent $j$ as follows:

$$
\operatorname{err}_{t}^{j}=\frac{1}{t} \sum_{\tau=1}^{t}\left|\frac{\tilde{S}_{j, \tau}}{\hat{S}_{j, \tau}}-1\right| \cdot 100
$$


$\widehat{S_{j}}$ is the approximated relative pseudo-Pareto weight that we obtain directly from our perturbation method. $\tilde{S}_{j}$ is constructed by dynamically updating our equilibrium recursion (i.e., an exact dynamic equation) for $S_{j, t}$ using our approximated forcing elements, $\hat{M}_{j, t}, \hat{M}_{1, t}, \hat{C}_{j, t} / \hat{C}_{j, t-1}$, and $\hat{C}_{1, t} / \hat{C}_{1, t-1}$. Examination of the discrepancy between $\tilde{S}_{j}$ and $\hat{S}_{j}$ constitutes a valid metric for assessing the quality of the approximation, as this discrepancy accounts for the persistent impact that the errors on our forcing terms can have on the relative pseudo-Pareto weights.

We also show percentiles of the one-period-ahead errors, which tend to be many orders of magnitude smaller. Across all cases, the approximation errors are small and comparable to those obtained under regular time-additive preferences, and thus are negligible for all practical purposes.

\section{REFERENCES}

Anderson, E. W. (2005), “The dynamics of risk-sensitive allocations." Journal of Economic Theory, 125 (2), 93-150. [311, 312, 314, 315, 316, 320, 332, 339, 340, 346]

Anderson, E. W., L. P. Hansen, and T. J. Sargent (2012), "Small noise methods for risksensitive/robust economies." Journal of Economic Dynamics and Control, 36 (4), 468500. [313]

Backus, D., C. Coleman, A. Ferriere, and S. Lyon (2016), "Pareto weights as wedges in two-country models.” Journal of Economic Dynamics and Control, 72 (Supplement C), 98-110. [312]

Backus, D. K., B. R. Routledge, and S. E. Zin (2005), "Exotic preferences for macroeconomists." NBER Macroeconomics Annual 2004, 19, 319-414. [311]

Backus, D. K., B. R. Routledge, and S. E. Zin (2008), “Who holds risky assets?” Working Paper. [312]

Baker, S. D. and B. R. Routledge (2017), “The price of oil risk.” Working Paper, Carnegie Mellon University and University of Virginia. [313]

Bansal, R., D. Kiku, I. Shaliastovich, and A. Yaron (2014), "Volatility, the macroeconomy and asset prices." Journal of Finance, 69 (6), 2471-2511. [313]

Bansal, R. and A. Yaron (2004), "Risks for the long run: A potential resolution of asset pricing puzzles.” Journal of Finance, 59, 1481-1509. [311]

Borovička, J. (2018), "Survival and long-run dynamics with heterogeneous beliefs under recursive preferences." Working Paper, NYU. [313]

Colacito, R. and M. Croce (2013), "International asset pricing with recursive preferences." Journal of Finance, 68 (6), 2651-2686. [312, 324, 327]

Colacito, R. and M. M. Croce (2012), "International robust disagreement." American Economic Review, 102 (3), 152-155. [312] 
Colacito, R., E. Ghysels, J. Meng, and W. Siwarasit (2016), "Skewness in expected macro fundamentals and the predictability of equity returns: Evidence and theory." The Review of Financial Studies, 20 (8), 2069-2109. [313]

Cole, H. and M. Obstfeld (1991), "Commodity trade and international risk sharing. How much do financial markets matter?” Journal of Monetary Economics, 28, 3-24. [330, 334]

Duffie, D., P. Geoffard, and C. Skiadas (1994), "Efficient and equilibrium allocations with stochastic differential utility." Journal of Mathematical Economics, 23, 133-146. [314]

Dumas, B., R. Uppal, and T. Wang (2000), “The global stability of efficient intertemporal allocations.” Journal of Economic Theory, 99, 240-259. [314]

Epstein, L. G. (1987), “The global stability of efficient intertemporal allocations.” Econometrica, 55, 329-355. [314]

Epstein, L. G. and S. E. Zin (1989), "Substitution, risk aversion, and the temporal behavior of consumption and asset returns: A theoretical framework." Econometrica, 57 (4), 937-969. [313, 314, 327, 328, 329, 331, 332, 333]

Geoffard, P. Y. (1996), "Discounting and optimazing: Capital accumulation as a variational minmax problem.” Journal of Economic Theory, 69, 53-70. [314]

Hansen, L. and T. J. Sargent (1995), “Discounted linear exponential quadratic Gaussian control.” IEEE Trans. Automatic Control, 40 (5), 968-971. [311, 314]

Hansen, L. and T. J. Sargent (2008), Robustness. Princeton University Press, Princeton, NJ. [312]

Kan, R. (1995), "Structure of Pareto optima when agents have stochastic recursive preferences.” Journal of Economic Theory, 66 (2), 626-631. [312, 316, 346]

Kubler, F. and K. Schmedders (2012), "Financial innovation and asset price volatility." American Economic Review, 102 (3), 147-151. [312]

Kuehn, L.-A. and O. Boguth (2013), “Consumption volatility risk.” Journal of Finance, 68 (6), 2589-2615. [313]

Lucas, R. and N. Stokey (1984), “Optimal growth with many consumers.” Journal of Economic Theory, 32, 139-171. [312, 316, 346]

Ma, C. (1993), "Market equilibrium with heterogenous recursive-utility-maximizing agents.” Economic Theory, 3, 243-266. [312, 315, 346]

Ma, C. (1996), “Corrigendum: Market equilibrium with heterogeneous recursive-utilitymaximizing agents.” Economic Theory, 7, 567-570. [315, 346]

Pohl, W., K. Schmedders, and O. Wilms (2017), "Higher-order effects in asset pricing models with long-run risks.” Working Paper. [313]

Rabitsch, K., S. Stepanchuk, and V. Tsyrennikov (2015), "International portfolios: A comparison of solution methods.” Journal of International Economics, 97, 404-422. [330] 
Sciubba, E. (2005), "Asymmetric information and survival in financial markets." Economic Theory, 25 (2), 353-379. [343, 344]

Segal, G., I. Shaliastovich, and A. Yaron (2015), "Good and bad uncertainty: Macroeconomic and financial market implications." Journal of Financial Economics, 117 (2), 369397. [313]

Tallarini, T. (2000), “Risk-sensitive real business cycles.” Journal of Monetary Economics, 45, 507-532. [311, 314, 324]

Tretvoll, H. (2018), "Real exchange rate variability in a two-country business cycle model." Review of Economic Dynamics, 27, 123-145. [312]

Tsyrennikov, V. (2012), "Heterogeneous beliefs, wealth distribution, and asset markets with risk of default." American Economic Review, 102 (3), 156-160. [312]

Co-editor Karl Schmedders handled this manuscript.

Manuscript received 16 May, 2014; final version accepted 3 April, 2018; available online 5 April, 2018. 